This is an author produced version of a paper published in Nonlinear Analysis: Theory, Methods \& Applications. This paper has been peer-reviewed but does not include the final publisher proof-corrections or journal pagination.

Citation for the published paper:

Overgaard, Niels. (2009). Application of variational inequalities to the moving-boundary problem in a fluid model for biofilm growth. Nonlinear Analysis: Theory, Methods \& Applications, vol. 70, issue 10, p. null

URL: http://hdl.handle.net/2043/2866

Publisher: null

This document has been downloaded from MUEP (https://muep.mah.se) / DIVA (https://mau.diva-portal.org). 


\title{
Application of variational inequalities to the moving-boundary problem in a fluid model for biofilm growth ${ }^{\star}$
}

\author{
Niels Chr. Overgaard \\ Applied Mathematics Group, School of Technology and Society, Malmö University, \\ SE-20506 Malmö, Sweden.
}

\begin{abstract}
We consider a moving-boundary problem associated with a limiting case of the fluid model for biofilm growth proposed by J. Dockery and I. Klapper, SIAM J. Appl. Math. 62(3), 2001. Concepts of classical, weak, and variational solutions for this problem are introduced. Classical solutions with radial symmetry are constructed, and estimates for their growth are given. Using a weighted Baiocchi transform it is shown that every classical solution is a weak solution. Every weak solution is in turn equivalent to the solution-set of a family of variational inequalities for an elliptic PDE (the variational solution). This allow us to show that, given arbitrary initial data at time $t=0$ (any bounded open set), there exists a weak solution defined for all times $t \geq 0$. Upper bounds for the weak solutions are given, and a semi-group property is proved. The background for this problem, and a model derivation is also presented.
\end{abstract}

Key words: Biofilm model, moving-boundary problem, variational inequality. 1991 MSC: 35R35, 49J10, 92B05

\footnotetext{
* This work was supported by the Swedish Knowledge Foundation (URL: http://www.kks.se/).

Email address: nco@ts.mah.se (Niels Chr. Overgaard).
} 


\section{Introduction}

Given a bounded open set $B \subset \mathbf{R}^{n}, n>0$ fixed, let $p_{B}$ denote the solution of the boundary value problem

$$
\left\{\begin{aligned}
(-\Delta+1) p_{B} & =1 \text { in } B \\
p_{B} & =0 \text { on } \partial B .
\end{aligned}\right.
$$

The purpose of this paper is to show that the following moving-boundary problem admits solutions in a certain weak sense.

(P) Given an initial bounded open set $B \subset \mathbf{R}^{n}$ and a number $T>0$. Find a set-valued function $[0, T] \ni t \mapsto B_{t} \subset \mathbf{R}^{n}$ such that (i) $B_{t}$ is bounded and open for any $0 \leq t \leq T$, (ii) $B_{0}=B$, and (iii) if $p_{t}=p_{B_{t}}$ is the solution of (1), then the free boundary $\Gamma_{t}=\partial B_{t}$ moves with a normal velocity $d \Gamma_{t} / d t$ given by,

$$
\frac{d}{d t} \Gamma_{t}=-\frac{\partial p_{t}}{\partial n}
$$

were $\partial / \partial n$ denotes the outward normal derivative on $\Gamma_{t}$.

This problem has its origin in mathematical (micro-)biology, where it arises as a limiting case in the biofilm model proposed by J. Dockery and I. Klapper [7]. The model describes the expansion of a bacterial colony under influence of an internal pressure ( $p_{B}$ above) caused by cell divisions inside the colony.

\subsection{What is a biofilm?}

Bacteria living in aqueous environments can be found in either planktonic form, where the individual bacteria are suspended in the bulk fluid, or as bacterial biofilms, which are colonies of bacteria attached to solid surfaces immersed in the liquid. The vast majority of all bacteria live in biofilms. In fact, bacteria in planktonic form are more often found on controlled laboratory environments than in nature.

Basically, a biofilm system consists of billions of bacteria adhering to a substratum, a thin conditioning film of proteins adsorbed to the solid surface. Nutrients, e.g. oxygen or ammonium, are brought to the individual bacteria of the biofilm by a combination of convection and diffusion in the bulk fluid, and by diffusion inside the biofilm. As a part of their metabolism, the bacteria excrete a slime of polymeric material. This extracellular polymeric substance (EPS) helps the bacteria to stick together and keeps the biofilm attached to 
the substratum. An average biofilm is about $300 \mu \mathrm{m}$ in thickness. This should be compared to the linear dimensions of a typical bacterium which is about 1 $\mu \mathrm{m}$. (See Figure 1.)

Biofilms are found almost everywhere; on ship hulls, in water pipes, on peoples teeth (as dental plaque), on medical implants, catheters and dialysis machines, just to give a few examples, and they have an enormous impact on our everyday lives. The presence of a biofilm can be damaging. On ship hulls or inside pipelines, biofilm coatings may result in reduced speed or loss of pressure, leading to increased costs for the operator. In medical devices or inside the human body, infections by bacterial biofilms may prove fatal. Moreover, once a biofilm has established itself, it may be hard to get rid of. The bacterial community inside a biofilm has proved to be remarkably resistant to chemical agents, antibiotics, and the human immune system. So, contaminating biofilms often have to be removed by mechanical means. For the biofilm on your teeth, the removal can be accomplished with the aid of a toothbrush, which sounds easy enough, but for medical implants, the only sure way to get rid of a biofilm infection is to remove the infected device entirely [28]. However, sometimes the presence of a biofilm may be beneficial. For instance, biofilms have been used in waste water treatment for more than 50 years.

\subsection{Biofilm Models}

Two decades ago it was generally believed that biofilms grow in homogeneous flat layers, and the mathematical biofilm models available at the time, e.g. [30], were essentially one-dimensional, with the biofilm growing in the direction perpendicular to the colonized surface. All this changed when confocal scanning laser microscopy was introduced to the biofilm research community in the early nineties. It was quickly realized that biofilms may sometimes develop elaborate geometric architectures, with valleys, channels, and fingering protuberances [5]. Nontrivial morphologies can of course not be captured in a one-dimensional model, and the following years saw several attempts to construct multi-dimensional biofilm models. Most of these models are either discrete, e.g [16], or semi-discrete [20,21,32,18], using cellular automata or similar discrete representations for the bacteria in the biofilm. Some continuum models have also emerged, most notably the reaction-diffusion system by Eberl at. al. [8], and the fluid model of Dockery and Klapper [7]. Existence and longtime behavior for the reaction-diffusion model [8] were investigated in [9]. In [7], the fluid model was solved in the (essentially one-dimensional) case of a plane biofilm interface. A linear stability analysis of this solution was carried out, showing that it admits fingering instabilities under certain circumstances. This indicates that the model is capable of producing solutions with complicated geometries, as observed in real biofilms. Questions about existence and 


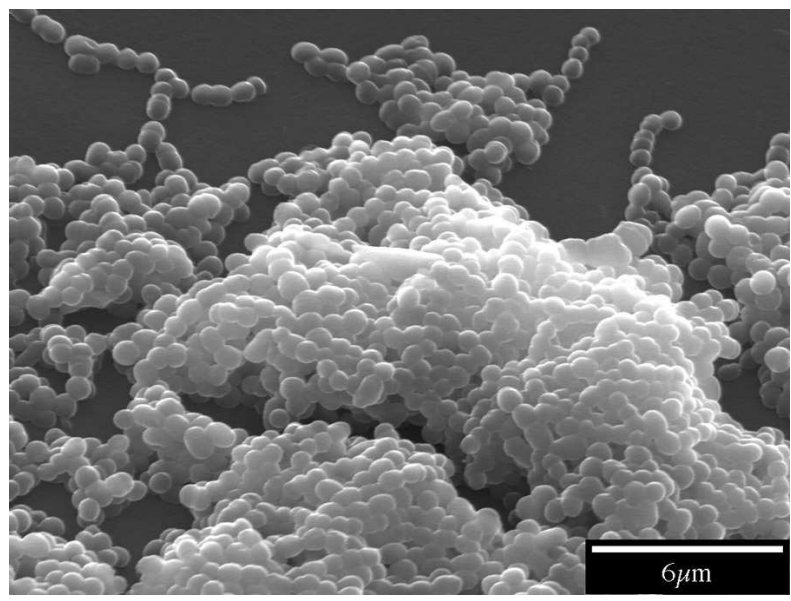

Fig. 1. A scanning electron microscope image of a one day old biofilm of the bacteria Streptococcus mutans. The biofilm is grown on a glass plate in a flow cell. Courtesy: Jessica Nielands at Oral Biology, Malmö University, and Dr Anne Meyer, Bob Forsberg at Center for Biosurfaces, University of Buffalo.

uniqueness of more general solutions to the model is, however, not discussed.

\subsection{Contributions and Organization of the Paper}

The goal of the present paper is to make a rigorous mathematical analysis of the problem $(\mathrm{P})$, which, as mentioned earlier, stems from the model in [7]. This problem has some similarity to a free boundary problem for Hele-Shaw flows, where a "blob" of fluid inside a laminar cell, called a Hele-Shaw cell [1], is made to expand by the injection of additional fluid at a fixed point inside the initial blob, the injection rate being known. One may think of $(\mathrm{P})$ as a Hele-Shaw problem where the injection takes place at every point of the blob, with rates that are not known in advance. The Hele-Shaw problem was (essentially) introduced by Richardson [23], and has later been taken up by several authors, most notably by Gustafsson [13], Elliott and Janovský [10], and Sakai [26]. See also [2,22]. Gustafsson's paper has been a major inspiration for the present work, and we have adapted many of the ideas therein to our problem. Perhaps, most importantly, the notion of weak solutions.

The paper contains what we believe are three main contributions. First of all, the moving-boundary problem $(\mathrm{P})$ is extracted from the original $\mathrm{D}-\mathrm{K}$ model as a limiting case. This problem is not only relevant to the biofilm community, but it is also interesting in its own right, as a mathematical problem. Secondly, the growth of the radial solutions are estimated by use of some properties of the Bessel functions which are not readily found in the literature. Thirdly, it is shown rigorously how to reformulate the moving-boundary problem as a variational inequality, by using a weighted Baiocchi transform. This result is 
used to prove existence and uniqueness of weak solutions of $(\mathrm{P})$. In view of the citation "... it is a red letter day when a free boundary problem can be cast into a variational formulation" [19, p. 334], the latter may be considered to be the main result in the paper.

The remainder of the paper is organized as follows: Section 2 gives a fairly detailed presentation of the biofilm model of Dockery and Klapper [7], henceforth called the $D-K$ model. Imposing the further hypotheses in $\S 2.2$, a simplified version of the model is obtained, which corresponds to the so-called growthlimited case. The simplified model is presented in dimension free form in $\S 2.3$, and the problem $(\mathrm{P})$ is finally obtained in $\S 2.4$. In $\S 3$, the notion of a classical solution of $(\mathrm{P})$ is defined, and classical solutions with radial symmetry are constructed. The growth of these solutions are estimated using a result (Proposition 3) proved in Appendix A. Section 4 contains a list of frequently used notation, and certain facts about Sobolev spaces. Moreover, differentiation and integration of (one-variable) functions with values in Banach spaces is recalled. In $\S 5$, the notion of a weak solution of $(\mathrm{P})$ is defined in a manner similar to that in Gustafsson [13]. We introduce a new dependent variable, by applying a weighted version of the classic Baiocchi transform to the pressure. This allow us to show (Theorem 6) that every classical solution of $(\mathrm{P})$ gives a weak solution of $(\mathrm{P})$. Finally, in $\S 6$ and $\S 7$, it is shown that every weak solution of $(\mathrm{P})$ corresponds to the solution set of a family of variational inequalities (called a variational solution of $(\mathrm{P})$ ), and vice versa. This immediately leads to the existence and uniqueness of weak solutions, using nothing more than the projection theorem for Hilbert spaces. The solutions exist for all positive times $t \geq 0$ (Theorem 16), and form a semi-group (Theorem 19), a fact that may be useful in numerical computations. Notice that whereas the first third of the paper is quite elementary, and to some extend standard, the latter two-thirds use more demanding methods from functional analysis.

\section{The Fluid Model of Dockery and Klapper}

The D-K model, which is inspired by tumor growth models such as [12], considers a biofilm system consisting of a liquid phase, the bulk fluid, and a biofilm, made up of a single bacterial species and EPS. The model assumes the bacteria feed on a single limiting nutrient, e.g. oxygen, and that the biomass can be treated as a homogeneous substance, whose properties are similar to that of an incompressible, viscous fluid, which is immiscible with the liquid phase, and obeys Darcy's law. Moreover, the bulk fluid closest to the biofilm, known as the boundary layer in the biofilm modelling literature (see $[21,18]$ ), is assumed to be in a state close to hydrostatic equilibrium, so that the fluid flow can be ignored. Outside the boundary layer there is a large reservoir of the nutrient, which diffuses freely through the boundary layer and into the biofilm, where 
it is consumed and transformed into new biomass by the bacteria, forcing the biofilm to expand.

\subsection{Mathematical Formulation of the Model}

Imagine that the system is contained in a open subset $\Omega \subset \mathbf{R}^{n}$ with boundary $\partial \Omega$. The dimension $n$ can be any positive integer, but only $n=1,2$, and 3 have direct relevance for biological applications. Assume the biofilm occupies a bounded open subset $B_{t}$ of $\Omega$ at time $t \geq 0$. Define $B_{t}^{\delta}=\left\{x \in \Omega\right.$ : $\operatorname{dist}\left(x, B_{t}\right)<$ $\delta$, where $\delta>0$. Then the set $B_{t}^{\delta} \backslash B_{t}$ is the boundary layer, and $\delta$ the boundary layer thickness.

Let $\rho=\rho(x, t)$ denote the density of the biofilm at $x \in \Omega$ and $t \geq 0$. Since the biomass is incompressible and immiscible with the bulk fluid, it follows that

$$
\rho(x, t)=\left\{\begin{array}{l}
\rho_{0} \text { for } x \in B_{t} \\
0 \text { for } x \in \Omega \backslash B_{t}
\end{array},\right.
$$

for some constant $\rho_{0}>0$, corresponding to the density of the biological material. Thus, the biomass is separated from the bulk fluid by a sharp interface, something that seems to be approximately true for real biofilms (c.f. [5, p. 138]). As we shall see later, the "state" of the biofilm at time $t$ is adequately described by the set $B_{t}$. Therefore, in order to describe the growth of the biofilm, it suffices to track the biofilm interface $\Gamma_{t}=\Omega \cap \partial B_{t}$ with respect to $t$.

The concentration of the (limiting) nutrient is denoted $c=c(x, t)$. The model assumes that diffusion is responsible for the transport of the nutrient, both in the boundary layer, and inside the biofilm. The bacteria in the biofilm consume the nutrient at a rate $r>0$ which we assume has the form

$$
r=r(\rho, c)=\rho f(c),
$$

where $f: \mathbf{R} \rightarrow \mathbf{R}$ is a given function called the reaction kinetics of the process. The consumed nutrient is transformed into new biomass at the same rate, by cell fission and excretion of EPS, hence $c$ solves a reaction-diffusion equation of the form

$$
\frac{\partial c}{\partial t}-\nabla \cdot\left(D_{0} \nabla c\right)=-r
$$

where $D_{0}>0$ is the diffusivity of the nutrient, which we assume is constant, for simplicity.

Since the biomass is incompressible, the addition of new biomass will lead to an expansion of the biofilm, hence the set $B_{t}$ changes over time. Consequently, 
the density (2), hence the right hand side of (3) are time-dependent. However, and this is crucial for the D-K model as well as most other multi-dimensional biofilm models, the growth process is much slower that the diffusion process, so the nutrient concentration is always close to equilibrium $(\partial c / \partial t \approx 0)$, see $[21,16,32,18]$. Therefore, the concentration $c$ is taken to be the steady-state solution of (3). In other words, it is assumed that the nutrient concentration $c=c(x, t)$ solves the following boundary value problem,

$$
\begin{cases}-D_{0} \Delta c+\rho f(c)= & \text { in } B_{t}^{\delta}, \\ c=c_{0} & \text { in } \Omega \backslash B_{t}^{\delta}, \\ \frac{\partial c}{\partial n}=0 & \text { on } \partial \Omega,\end{cases}
$$

The first boundary conditions states that the nutrient has a fixed concentration $c=c_{0}$ outside the boundary layer. The second one states that nutrients are not allowed to penetrate through the walls of the container. Notice that the biofilm density $\rho$ makes a jump across the interface $\Gamma_{t}$, so the PDE has discontinuous coefficients.

The D-K model assumes the biomass behaves like a viscous, incompressible fluid whose volumetric flow, described here by the vector field $\mathbf{u}=\mathbf{u}(x, t)$, obeys Darcy's law:

$$
\mathbf{u}=-\lambda \nabla p
$$

where $p=p(x, t)$ is the pressure, and $\lambda>0$ is a constant. The flux of biomass in $B_{t}$ is $\rho_{0} \mathbf{u}$, and the rate at which new biomass is created is $r=\rho_{0} f(c)$. Conservation of mass therefore requires that $\operatorname{div}\left(\rho_{0} \mathbf{u}\right)=\rho_{0} f(c)$ in $B_{t}$ (Equation of continuity). Using Darcy's law (5) we find that the pressure solves

$$
\begin{cases}-\lambda \Delta p-f(c)=0 & \text { in } B_{t} \\ \frac{\partial p}{\partial n}=0 & \text { on } \partial \Omega \\ p=0 & \text { on } \Gamma_{t}=\Omega \cap \partial B_{t} .\end{cases}
$$

Here the first boundary condition means that biomass is not allowed to pass through the walls of the container, and the second one follows from a physical consideration: The viscosity of the surrounding liquid is much smaller than that of the biomass, so the pressure gradient needed in order for the bulk fluid to adapt to the expanding biofilm is very small, hence the pressure outside the biofilm is nearly constant. This is idealized in the model by assuming that $p$ is identically zero in the bulk fluid.

Finally, in order to account for the added biomass, the interface $\Gamma_{t}$ has to move with a speed equal to the normal component of the volumetric flow in 
(5), that is

$$
\frac{d}{d t} \Gamma_{t}=\mathbf{u} \cdot \mathbf{n}=-\lambda \frac{\partial p}{\partial n} \quad \text { on } \Gamma_{t}
$$

where $\mathbf{n}$ is the outward unit normal to $B_{t}$, and the symbol $d \Gamma_{t} / d t$ denotes the normal velocity of the surface. (Defined in $\S 3.1$.)

Now the dynamics of the D-K model is completely described. If the biofilm initially occupies the bounded, open set $B$, then we are requested to solve the following problem:

(P*) Given a number $T>0$, find a set-valued function $t \mapsto B_{t}$ such that (i) $B_{t} \subset \Omega$ is bounded and open for $0 \leq t \leq T$, (ii) $B_{0}=B$, and (iii) if the nutrient concentration $c$ is the solution of (4), and the pressure $p$ solves (6), then the normal velocity of the biofilm interface $\Gamma_{t}$ satisfies $(7)$.

Remarks: 1. If the reaction kinetics $f$ is a non-decreasing and (uniformly) Lipschitz continuous function, then the boundary value problem (4) has a unique solution (in the weak sense). In biological applications it is often the case that $f(0)=0$. (When the nutrient concentration is zero there is nothing to eat!) If this condition is imposed on $f$, then it follows from the maximum principle that the solution satisfies $c>0$, as required by physical considerations. Among biologists and chemical engineers, a popular choice of reaction kinetics is the function

$$
f(c)=\left\{\begin{array}{cr}
k_{1} c /\left(k_{2}+c\right), & \text { for } c>0 \\
0, & \text { otherwise }
\end{array}\right.
$$

where $k_{1}, k_{2}>0$ are fixed constants. This is known either as the Monod- or the Michäelis-Menten kinetics. Linear (or first order) kinetics $f(c)=a c, a>0$, and zero-order kinetics $f(c)=a, a>0$, are other common choices.

2. In fluid mechanics, Darcy's law occurs in the modelling of fluid flows in porous media [6, p.32], and in the so-called Hele-Shaw flow (or thin film approximation). The latter refers to the flow $\mathbf{u}$ of an incompressible viscous fluid trapped inside a narrow gap between two parallell plates. Such a system is essentially two-dimensional, and the Navier-Stokes' equations for $\mathbf{u}$ reduce to $\operatorname{div} \mathbf{u}=0$ and (5), see [1, p.238]. The constant $\lambda$ in the Hele-Shaw flow is proportional to the gap-width, and inversely proportional to the viscosity of the fluid. 


\subsection{Simplifying Hypotheses}

Three simplifications are made to the original D-K model, which will eventually lead us to the problem (P).

$\left(\mathrm{H}_{1}\right)$ We assume that the boundary layer thickness is zero, $\delta=0$. This implies that the nutrient concentration has its reservoir strength $c_{0}$ up to the biofilm interface $\Gamma_{t}$. This corresponds to a situation sometimes referred to as the growth-limited case in the biofilm literature [20, p. 109], and is relevant in modelling of so-called moving bed biofilm reactors. Observe that with this hypothesis, the biofilm density is constant $\rho=\rho_{0}$ in (4).

$\left(\mathrm{H}_{2}\right)$ We consider linear reaction kinetics: $f(c)=a c$, where $a>0$ is constant. (In $\S 8$, zero order kinetics is briefly considered.)

$\left(\mathrm{H}_{3}\right)$ Finally, we take $\Omega=\mathbf{R}^{n}$, that is, we consider a "free" biofilm. Our main reason for restricting the analysis to this special case is to avoid some technical difficulties associated with the presence of a complicated boundary $\partial \Omega$, when proving that each classical solution of $(\mathrm{P})$ gives rise to a weak solution of $(\mathrm{P})$. In this case $\partial \Omega$ is empty, so $\Gamma_{t}=\partial B_{t}$, and the Neumann boundary conditions for $p$ and $c$ becomes irrelevant.

\subsection{Dimension Free Formulation}

In order to write the $\mathrm{D}-\mathrm{K}$ model in dimension free form, we first list the physical dimensions of the model variables. If $q$ is some physical quantity, then we use the notation $[q]$ to denote its dimension. In our case, the dimensions of all the model variables can be expressed in terms of length $L$, mass $M$, and time $T$. From now on the hypotheses $\left(\mathrm{H}_{1}\right)-\left(\mathrm{H}_{3}\right)$ are imposed. Therefore the relevant physical quantities of the $\mathrm{D}-\mathrm{K}$ model are:

$$
[p]=\frac{M}{T^{2} L}, \quad[\lambda]=\frac{T L^{3}}{M}, \quad[\rho]=\frac{M}{L^{3}}, \quad[c]=\frac{M}{L^{3}}, \quad\left[D_{0}\right]=\frac{L^{2}}{T}, \quad[a]=\frac{1}{T} .
$$

Notice that $[\rho]=[c]$ and $\left[\sqrt{D_{0} / a}\right]=L$. If we introduce the following new dependent variables

$$
\tilde{c}=\frac{c}{c_{0}} \quad \text { and } \quad \tilde{p}=\frac{\lambda p}{c_{0} D_{0} / \rho_{0}},
$$

and the following new independent variables

$$
\tilde{t}=\frac{t}{\rho_{0} / a c_{0}} \quad \text { and } \quad \tilde{x}=\frac{x}{\sqrt{D_{0} / a}}
$$


then (4) and (6) become (after the tildes have been dropped):

$$
\left\{\begin{array}{ccc}
-\Delta c+c=0 & \text { and }-\Delta p-c=0 & \text { in } B_{t} \\
c=1 & \text { and } \quad p=0 & \text { on } \Gamma_{t}
\end{array}\right.
$$

Moreover, the normal velocity (7) of the biofilm interface becomes

$$
\frac{d}{d t} \Gamma_{t}=-\frac{\partial p}{\partial n} \quad \text { on } \Gamma_{t}
$$

Notice the remarkable fact that, after the equations have been rewritten in dimension free form, there are no free parameters left in the model.

\subsection{Elimination of the Nutrient Concentration}

Perhaps the reader has already observed that when $\delta=0$ the nutrient concentration can be eliminated from the problem. In fact, if the PDEs for $c$ and $p$ in (9) are added, then

$$
-\Delta(c+p)=0 \quad \text { in } B_{t}, \quad c+p=1 \quad \text { on } \Gamma_{t},
$$

so, by the maximum principle, $c+p=1$ in all of $B_{t}$. If this result is reintroduced into the PDE for the pressure, then the problem $\left(\mathrm{P}^{*}\right)$ in dimension free form, that is, with (9) and (10) instead of (4)-(6), becomes the problem (P) in $\S 1$.

\section{Classical Solutions and Examples}

In this section, the notion of a classical solution to the problem $(\mathrm{P})$ is defined, and the classical solutions with radial symmetry is constructed in each dimension $n \geq 1$. The growth of the radial solutions is analyzed, leading to easy upper and lower bounds on the size of $B_{t}$, and insight into the long-time behavior of the solutions.

Before the definition of a classical solution can be given, analytical descriptions of the moving biofilm interface and its normal velocity are needed.

\subsection{Classical Solutions in Implicit Representation}

Let $\mathcal{B}$ denote the class of bounded open subsets of $\mathbf{R}^{n}$. We are interested in sets $B \in \mathcal{B}$ whose boundaries, $\Gamma=\partial B$, have certain regularity properties. 
A convenient way to single out such sets is to use an implicit representation, that is, to require the existence of a smooth function $f: \mathbf{R}^{n} \rightarrow \mathbf{R}$ such that $B=\{x: f(x)<0\}$. In fact, if $f \in C^{k}\left(\mathbf{R}^{n}\right), k \geq 1$, and zero is a regular value of $f$ (i.e. $f(x)=0$ implies $\nabla f(x) \neq 0$ ) then $\Gamma=\{x: f(x)=0\}$ is a $C^{k}$-surface, by the implicit function theorem. We may always assume that $f$ is a proper function, that is, $f^{-1}(K)$ is compact for every compact $K \subset \mathbf{R}$, in other words, $|f(x)| \rightarrow \infty$ as $|x| \rightarrow \infty$.

The implicit representation can be modified so as to include moving boundaries: If $I=[a, b]$ is a time-interval and $f \in C^{k}\left(\mathbf{R}^{n} \times I\right)$ is a proper function such that zero is a regular value of $f(\cdot, t): \mathbf{R}^{n} \rightarrow \mathbf{R}$, for all $t$, then $I \ni t \mapsto B_{t}:=\{x: f(x, t)<0\} \in \mathcal{B}$ is a well-defined set-valued function, and $t \mapsto \Gamma_{t}:=\{x: f(x, t)=0\}$ the corresponding moving boundary.

Next, the definition of the normal velocity $d \Gamma_{t} / d t$ of a moving boundary is recalled. Assume $0 \in I$ and $x_{0} \in \Gamma_{0}$. Suppose a particle, whose motion is described by a differentiable curve $\alpha: I \rightarrow \mathbf{R}^{n}$, is following the moving boundary $\Gamma_{t}$, and is passing through the point $x_{0}$ at $t=0$. Then $\alpha(0)=x_{0}$, and differentiation of the identity $f(\alpha(t), t)=0$ with respect to time yields

$$
\dot{\alpha}(0) \cdot \mathbf{n}\left(x_{0}\right)=-\frac{\partial f\left(x_{0}, 0\right) / \partial t}{\left|\nabla_{x} f\left(x_{0}, 0\right)\right|}
$$

where $\dot{\alpha}$ is the velocity of the particle, and $\mathbf{n}$ the outward unit normal to $B_{0}$. Now, the left-hand side of this equation is the normal component of the particle's velocity at $t=0$. This quantity is independent of the particular choice of curve $\alpha$ passing through the point $x_{0}$ at $t=0$, because the righthand side of (11) makes no reference to $\alpha$. Similarly, it is also independent of the particular choice of the function $f$ representing $\Gamma_{t}$, because $f$ does not appear in the left-hand side of (11). In conclusion, the quantity (11) describes an intrinsic property of the moving surface. Therefore the normal velocity of $\Gamma_{t}$ at time $t$ is defined as the function $d \Gamma_{t} / d t: \Gamma_{t} \rightarrow \mathbf{R}$ given by

$$
\frac{d}{d t} \Gamma_{t}(x):=-\frac{\partial f(x, t) / \partial t}{\left|\nabla_{x} f(x, t)\right|}, \quad\left(x \in \Gamma_{t}\right) .
$$

The following notion of a classical solution is more or less standard:

Definition 1 Given a set $B \in \mathcal{B}$ and a number $T>0$. A set-valued mapping $[0, T] \ni t \mapsto B_{t} \in \mathcal{B}$ is called a classical solution of $(\mathrm{P})$ with initial data $B$ if there exists a proper function $f \in C^{2}\left(\mathbf{R}^{n} \times[0, T]\right)$ such that

(a) zero is a regular value for $f(\cdot, t): \mathbf{R}^{n} \rightarrow \mathbf{R}$ for all $t \in[0, T]$,

(b) $B_{t}=\{x: f(x, t)<0\}$ for all $t \in[0, T]$, 
(c) and, if $p_{t}(x)=p(x, t)$ solves the boundary value problem:

$$
\left\{\begin{aligned}
(-\Delta+1) p_{t} & =1 \text { in } B_{t}, \\
p_{t}=0 & \text { on } \Gamma_{t}=\partial B_{t} .
\end{aligned}\right.
$$

then the moving-boundary condition:

$$
\nabla_{x} f \cdot \nabla_{x} p_{t}-\frac{\partial f}{\partial t}=0 \quad \text { on } \Gamma_{t}
$$

holds for all $t \in[0, T]$.

Remarks: 1 . The moving-boundary condition (14) is, in view of our definition (12) of the normal velocity, equivalent to the one in $(\mathrm{P})$. Since $\Gamma_{t}=\{x$ : $f(x, t)=0\}$ is of class $C^{2}$, the pressure satisfies $p_{t} \in C^{2}\left(B_{t}\right) \cap C^{1}\left(\overline{B_{t}}\right)$, see $[11$, Thm. 8.34]. Therefore $\nabla p_{t}$ is defined on $\Gamma_{t}$, so the moving-boundary condition (14) makes sense.

2. By the strong maximum principle $p_{t}>0$ in $B_{t}$, hence $\partial p_{t} / \partial n<0$ on the moving boundary $\Gamma_{t}$ (Hopf's Lemma). This implies that a classical solution $t \mapsto B_{t}$ of $(\mathrm{P})$ is monotonously increasing with respect to inclusion $\left(B_{s} \subset B_{t}\right.$ for $s \leq t)$.

3. Our definition of a classical solution is rather restrictive. For instance, it is impossible for $B_{t}$ to change topology because of the condition on $f$ 's gradient on the moving boundary. So, if $B_{0}$ consists of two separate connected components which expand and meet at a later time $t_{0}$, then a classical solution with initial set $B_{0}$ cannot be defined beyond the time $t_{0}$. However, Definition 1 suffices for our purposes; to construct radially symmetric classical solutions, and to motivate the notion of a weak solution of $(\mathrm{P})$, introduced in $\S 5$.

\subsection{Radial Solutions}

Fix an integer $n \geq 1$, a number $R_{0}>0$, and set $B_{0}=\left\{x \in \mathbf{R}^{n}:|x|<\right.$ $\left.R_{0}\right\}$. We seek a radially symmetric classical solution of $(\mathrm{P})$ with initial data $B_{0}$. More precisely, we want to determine a function $R=R(t)$ such that $B_{t}=\{x:|x|<R(t)\}$. The solution is described implicitly by the function $f(x, t)=\left(|x|^{2}-R(t)^{2}\right) / 2$, and the pressure can be written in the form $p(x, t)=$ $g(r ; R(t))$, where $r=|x|$.

We first determine the function $g(r)=g(r, R)$ for a fixed, but arbitrary, positive value of $R$. Clearly $g$ must satisfy $g(R)=0$, and in order for the pressure to be smooth at the origin, also $g^{\prime}(0)=0$. Therefore (13) reduces to 
the following boundary value problem

$$
\left\{\begin{array}{l}
-g^{\prime \prime}(r)-\frac{n-1}{r} g^{\prime}(r)+g(r)=1 \quad \text { for } 0<r<R \\
g^{\prime}(0)=0, \quad g(R)=0 .
\end{array}\right.
$$

The solution of this problem can be expressed in terms of Bessel functions of the first kind:

$$
J_{\nu}(z)=\sum_{k=0}^{\infty} \frac{(-1)^{k}\left(\frac{1}{2} z\right)^{\nu+2 k}}{k ! \Gamma(\nu+k+1)} .
$$

In fact, defining

$$
G_{\nu}(z)=(i z)^{-\nu} J_{\nu}(i z)
$$

we see from (16) that $G_{\nu}$ is an entire analytic function, and it is easily checked that the solution of $(15)$ is

$$
g(r)=g(r ; R)=1-\frac{G_{\nu}(r)}{G_{\nu}(R)} \quad\left(\nu=\frac{n}{2}-1\right) .
$$

By definition, $\nabla_{x} f=x, \partial f / \partial t=-R(t) R^{\prime}(t)$, and $\nabla_{x} p_{t}=g^{\prime}(r, R(t)) x / r$, so the moving-boundary condition (14) takes the following form:

$$
R(t)\left[g^{\prime}(R(t) ; R(t))+R^{\prime}(t)\right]=0 .
$$

Using (18) and $R(t)>0$, we find that $R$ must solve the initial value problem,

$$
R^{\prime}(t)=\frac{G_{\nu}^{\prime}(R(t))}{G_{\nu}(R(t))}, \quad R(0)=R_{0}, \quad\left(\nu=\frac{n}{2}-1\right)
$$

Since this problem has a unique solution defined for all $t \geq 0$ we have:

Proposition 2 The map $\mathbf{R}_{+} \ni t \mapsto B_{t}=\left\{x \in \mathbf{R}^{n}:|x|<R(t)\right\}$, where $R(t)$ is the solution of the initial value problem (19), is a classical solution of $(P)$ with initial data $B_{0}=\left\{x:|x|<R_{0}\right\}$. The corresponding pressure $p(x, t)=g(r ; R(t))$ is given by $(18)$.

When $n$ is an odd integer, the Bessel function $J_{\nu}, \nu=\frac{n}{2}-1$, can be expressed in terms of elementary functions, and the same is true for the solution of (15). For instance, if $n=1$ or $n=3$ the solutions are

$$
g(r ; R)=1-\frac{\cosh r}{\cosh R}, \quad \text { and } \quad g(r ; R)=1-\frac{(\sinh r) / r}{(\sinh R) / R} \quad(0<r<R) \text {, }
$$

respectively. For $n=1$ the initial value problem (19) has the solution

$$
R(t)=t+\ln \left[\sinh R_{0}+\left(\mathrm{e}^{-2 t}+\sinh ^{2} R_{0}\right)^{1 / 2}\right] .
$$


Since $y \mapsto \ln \left[y+\sqrt{1+y^{2}}\right]$ is the inverse of $\sinh x$ it readily follows that

$$
R(t) \leq t+R_{0}, \quad(t \geq 0) .
$$

Moreover, $R^{\prime}(t)$ is increasing, and tends to 1 as $t \rightarrow \infty$. The first observation is an easy bound on the growth of the solution, and the second one shows that the biofilm interface approaches a limiting speed monotonically as the biofilm grows larger. For $n=3$ the corresponding differential equation,

$$
R^{\prime}=\frac{\cosh R}{\sinh R}-\frac{1}{R}, \quad R(0)=R_{0},
$$

is more difficult. Instead of solving it, we observe that the right hand side of the differential equation is an increasing function which tends to 1 as $R \rightarrow \infty$, hence $R^{\prime}(t)<1$, and it follows that the estimate (20) holds even for $n=3$. Again, the speed of the moving boundary tends to one as the radius of $B_{t}$ tends to infinity. These observations are valid for the radial solutions in any number of dimensions, which is an easy consequence of the following result, whose proof is given in the appendix A.

Proposition 3 For $\nu>-\frac{1}{2}$ the function $G_{\nu}^{\prime}(r) / G_{\nu}(r), r \in \mathbf{R}$, is strictly monotonically increasing, $G_{\nu}^{\prime}(0) / G_{\nu}(0)=0$, and $\lim _{r \rightarrow \pm \infty} G_{\nu}^{\prime}(r) / G_{\nu}(r)= \pm 1$.

This has the following implications for the growth of the radially symmetric classical solutions constructed above.

Corollary 4 Let $B_{t}=\{x:|x|<R(t)\}$ be the radially symmetric classical solution of $(P)$ with initial data $B_{0}=\left\{x:|x|<R_{0}\right\}$. Then (a), the radius $R(t)$ is strictly increasing, tends to infinity as $t \rightarrow \infty$, and satisfies the bound (20). (b), the growth rate $R^{\prime}(t)$ is strictly increasing, and $R^{\prime}(t) \rightarrow 1$ as $t \rightarrow \infty$.

PROOF. $R(t)$ solves 19 , so (a) is an easy consequence of the bound $0<$ $G_{\nu}^{\prime}\left(R_{0}\right) / G_{\nu}\left(R_{0}\right) \leq R^{\prime}(t) \leq 1$, whereas (b) follows from (19) and the proposition, in view of (a).

\section{Notation}

Before proceeding with the analysis of $(\mathrm{P})$ in a more functional analytical setting, we give a list of frequently used symbols, and recall some facts about Sobolev spaces, and certain notions of derivatives and integrals of one-variable functions with values in Banach spaces.

Notation: $B-\mathrm{A}$ bounded open subset of $\mathbf{R}^{n}$. $B(x ; R)$-The open ball with center $x \in \mathbf{R}^{n}$ and radius $R>0$. 
$\mathcal{B}$ - The class of bounded open subsets of $\mathbf{R}^{n}$.

$B_{t}$-The bounded open subset occupied by the biofilm at time $t \geq 0 ; B_{t} \in \mathcal{B}$.

$\chi_{D}$ - The characteristic function of a subset $D \subset \mathbf{R}^{n}$;

$$
\chi_{D}(x)=\left\{\begin{array}{l}
1 \text { if } x \in D \\
0 \text { if } x \in \mathbf{R}^{n} \backslash D
\end{array} .\right.
$$

$\mathcal{X}=\left\{\chi_{B}: B \in \mathcal{B}\right\}$ - The characteristic functions corresponding to elements of $\mathcal{B}$.

$\nabla u=\left(\partial u / \partial x_{1}, \ldots, \partial u / \partial x_{n}\right)$ - The gradient of $u=u(x)$.

$u_{t}$-The function $u=u(x, t)$ when $t$ is regarded as a parameter.

$\nabla_{x} u$-The gradient of $u=u(x, t)$ with respect to the $x$-variables, i.e. $\nabla_{x} u=$ $\nabla u_{t}$.

$-\Delta u=-\sum_{i=1}^{n} \partial^{2} u / \partial x_{i}$ - The Laplacian of $u$.

supp $u$-The support of $u$, defined in the sense of distributions. (See e.g. [17].) $\eta, \eta^{*}$ - Cut-off functions. By a cut-off function we mean a differentiable function $\eta \in C_{0}^{\infty}$, such that $0 \leq \eta \leq 1$.

Function Spaces: $C_{0}^{k}(\Omega)$ - The space of $k$ times continuously differentiable functions with compact support in $\Omega ; C_{0}^{\infty}(\Omega)$ is the test functions, and $C_{0}^{\infty}=$ $C_{0}^{\infty}\left(\mathbf{R}^{n}\right)$.

$L^{p}=L^{p}\left(\mathbf{R}^{n}\right), 1 \leq p<\infty$-The Lebesgue spaces; $\|u\|_{L^{p}}=\left(\int_{\mathbf{R}^{n}}|u|^{p} d x\right)^{1 / p}$ is the norm of $u \in L^{p}$.

$W_{0}^{k, p}(\Omega)$ - The Sobolev spaces as defined in [4]. For brevity, we write $H_{0}^{k}(\Omega)=$ $W_{0}^{k, 2}(\Omega)$ and $H_{0}^{k}=H_{0}^{k}\left(\mathbf{R}^{n}\right)$. The Hilbert space $H_{0}^{1}$ is equipped with the inner product

$$
(u, v)_{H_{0}^{1}}=\int_{\mathbf{R}^{n}} \nabla u \cdot \nabla v+u v d x, \quad\left(u, v \in H_{0}^{1}\right),
$$

and the norm $\|u\|_{H_{0}^{1}}=(u, u)_{H_{0}^{1}}^{1 / 2}$.

$H^{-1}(\Omega)$ - The dual of $H_{0}^{1}(\Omega), H^{-1}=H^{-1}\left(\mathbf{R}^{n}\right)$. Elements in $H^{-1}(\Omega)$ may be identified with distributions in $\Omega$ of the form $v=f_{0}+\sum_{i=1}^{n} \partial f_{i} / \partial x_{i}$ (distributional derivatives), where $f_{0}, f_{1}, \ldots, f_{n} \in L^{2}$. We are often going to use the fact that the differential operator,

$$
-\Delta+\lambda: H_{0}^{1}(\Omega) \rightarrow H^{-1}(\Omega),
$$

is an isomorphism for $\lambda>0$, see [29, Chapter III]. If $\Omega$ is bounded, this assertion holds even for $\lambda=0$, i.e. for the Laplacian.

$\langle u, v\rangle$ - The duality paring between elements $u \in H_{0}^{1}(\Omega)$ and $v \in H^{-1}(\Omega)$. If $u$ and $v$ are well-behaved functions, then the duality bracket reduces to a Lebesgue integral, e.g.

$$
\langle u, v\rangle=\int_{\Omega} u v d x, \quad\left(u \in L^{p}, v \in L^{q}, \frac{1}{p}+\frac{1}{q}=1\right) .
$$


Derivatives and Integrals of Vector-Valued Functions: Let $X$ be a Banach space, and $X^{*}$ its dual. The pairing between a vector $x$ in $X$ and a linear functional $x^{*}$ in $X^{*}$ is denoted $\left\langle x^{*}, x\right\rangle$. A vector-valued function $[0, T] \ni t \mapsto$ $x_{t} \in X$ is said to be $w$-differentiable on $(0, T)$ if it is differentiable in the weak topology, i.e. if the function $t \mapsto\left\langle x^{*}, x_{t}\right\rangle$ is differentiable on $(0, T)$, for every $x^{*} \in X^{*}$, and the derivative satisfies

$$
\frac{d}{d t}\left\langle x^{*}, x_{t}\right\rangle=\left\langle x^{*}, w_{t}\right\rangle
$$

for some function $[0, T] \ni t \mapsto w_{t} \in X$. The vector $w_{t}$, called the $w$-derivative of $x_{t}$, is denoted $(d / d t) x_{t}=w_{t}$.

Suppose the vector-valued function $[0, T] \ni t \mapsto x_{t} \in X$ is continuous, then there exists a unique vector $w \in X$ such that $\left\langle x^{*}, w\right\rangle=\int_{0}^{T}\left\langle x^{*}, x_{t}\right\rangle d t$ for all $x^{*} \in X^{*}$, see Rudin [25, Chapter 3]. The vector $w$ is called the integral of $x_{t}$, and it is denoted

$$
w=\int_{0}^{T} x_{t} d t .
$$

The vector-valued integral has many of the usual properties associated with integrals; it is clearly linear, and $\left\|\int_{0}^{T} x_{t} d t\right\| \leq \int_{0}^{T}\left\|x_{t}\right\| d t$. Moreover, if $A$ : $X \rightarrow Y$ is a continuous linear mapping into another Banach space $Y$, then $t \mapsto A x_{t}$ is continuous, and

$$
\int_{0}^{T} A x_{t} d t=A\left(\int_{0}^{T} x_{t} d t\right),
$$

where the left-hand side is well-defined as a vector-valued integral in $Y$.

\section{Weak Solutions}

We are going to consider a certain kind of weak solutions of $(\mathrm{P})$, where we focus on the characteristic function $\chi_{t}:=\chi_{B_{t}}$ instead of $B_{t}$ itself. Let $\mathcal{X}$ denote the class of characteristic functions of bounded open subsets of $\mathbf{R}^{n}$,

$$
\mathcal{X}=\left\{\chi_{B}: B \in \mathcal{B}\right\} .
$$

Following [13] we now introduce:

Definition 5 A mapping $[0, T] \ni t \mapsto \chi_{t} \in \mathcal{X}$ is called a weak solution of $(\mathrm{P})$ with initial data $\chi_{0}$ if the vector-valued function $[0, T] \ni t \mapsto u_{t} \in H_{0}^{1}$, where $u_{t}$ defined by the equation

$$
\left.(-\Delta+1) u_{t}=\mathrm{e}^{t} \chi_{0}-\chi_{t}, \quad \text { (Equality in } H^{-1}\right)
$$


satisfies the following conditions for every $t \in[0, T]$,

$$
\begin{gathered}
u_{t} \geq 0, \\
\left\langle u_{t}, \eta-\chi_{t}\right\rangle=0 .
\end{gathered}
$$

for all cut-off functions $\eta \geq \chi_{t}$.

Remarks: 1. $u_{t} \in H_{0}^{1}$ is well-defined because the operator $-\Delta+1: H_{0}^{1} \rightarrow H^{-1}$ is an isomorphism, and the right member of (21a) belongs to $L^{2}$.

2. By monotone convergence, the condition (21c) implies that $\int u_{t}\left(1-\chi_{t}\right) d x=$ 0 , so $\operatorname{supp} u_{t} \subset \operatorname{supp} \chi_{t}$. Unfortunately, it is not possible to express this identity directly, using the duality bracket $\langle\cdot, \cdot\rangle$, because 1 , hence $1-\chi_{t}$, does not belong to $H^{-1}$.

3. The notion of a weak solution to (P) may seem a bit intricate at first, but it is in fact quite natural. First of all, we get rid of the implicit representation of $B_{t}$ by a function $f$ which is not unique. Instead the characteristic functions $\chi_{t}$ of are used. secondly, the notion of a solution is generalized in the sense that if $t \mapsto B_{t}$ is a classical solution of $(\mathrm{P})$ then $t \mapsto \chi_{t}:=\chi_{B_{t}}$ is a weak solution of (P). (See Theorem 6.) This is remarkable because the definition of weak solutions does mot make explicit reference to a moving-boundary condition on the free boundary. Exactly for this reason, weak solutions are a more flexible tool than classical solutions.

From now on, and the rest of this section, let $[0, T] \ni t \mapsto B_{t} \in \mathcal{B}\left(\mathbf{R}^{n}\right)$ be a classical solution of $(\mathrm{P})$, with the corresponding pressure $p_{t}$ and implicit representation by a function $f$, as in Definition 1.

In the definition of a classical solution, the pressure $p_{t}$ is defined on the set $B_{t}$ only. However, since the pressure is zero on the free boundary, it is natural to consider the extension of $p_{t}$ obtained by setting the pressure equal to zero outside $B_{t}$ :

$$
\bar{p}_{t}(x)=\left\{\begin{array}{cc}
p_{t}(x) & \text { for } x \in B_{t}, \\
0 & \text { otherwise. }
\end{array}\right.
$$

It is easy to see that $\bar{p}_{t} \in H_{0}^{1}$ for all $t \in[0, T]$. In fact, $p_{t} \in C^{1}\left(\bar{B}_{t}\right)$, by the remark after Definition 1 , and $p_{t}=0$ on $\partial B_{t}$, so the claim follows from a standard result in the theory of Sobolev spaces, see Brézis [4, Proposition IX.18]. Our aim is to prove the following result:

Theorem 6 Suppose $[0, T] \ni t \mapsto B_{t} \in \mathcal{B}\left(\mathbf{R}^{n}\right)$ is a classical solution of $(P)$. Let $u_{t}$ be the function given by the vector-valued integral

$$
u_{t}=\int_{0}^{t} \mathrm{e}^{t-s} \bar{p}_{s} d s \quad(0 \leq t \leq T),
$$


where $\bar{p}_{t}$ is the defined by (22). Then $u_{t} \in H_{0}^{1}$, and $[0, T] \ni t \mapsto \chi_{t}:=\chi_{B_{t}} \in \mathcal{X}$ is a weak solution of $(P)$ represented by the mapping $[0, T] \ni t \mapsto u_{t} \in H_{0}^{1}$.

Remark: The function $u_{t}$ is called the (weighted) Baiocchi transform of $\bar{p}_{t}$. At first the author believed the transform (23) to be new, however Gustafsson [15] has used essentially the same transform to solve a moving-boundary problem for a Hele-Shaw flow where a blob of fluid is "squeezed" between two parallell plates. This problem, by the way, is similar to the $\mathrm{D}-\mathrm{K}$ model with zero-order kinetics. (See $\S 8$.)

The hard part in the proof of this theorem is to show that (23) is a well-defined vector-valued integral, in the sense introduced in $\S 4$. To do this, we must show that $t \mapsto \bar{p}_{t}$ is a continuous mapping $[0, T] \rightarrow H_{0}^{1}$, which is the object of the next couple of lemmas.

Lemma 7 If $\chi$ (without an index) denotes the characteristic function of the set $B=\{(x, t): f(x, t)<0\}$, then the distributional $t$-derivative of $\chi$ satisfies

$$
\partial_{t} \chi=-\frac{\partial f / \partial t}{\left|\nabla_{x} f\right|} d s_{t} d t
$$

where $d s_{t}$ denotes the Euclidean surface measure on $\Gamma_{t}$, and $d t$ is the Lebesgue measure on the interval $[0, T]$.

PROOF. It is well-known that $\partial_{t} \chi=-N_{t} d s$, where $N_{t}$ is the $t$-component of the outward unit normal $N=\left(N_{x}, N_{t}\right)$ of $B$, and $d s$ is the Euclidean surface measure on $\Gamma=\{(x, t): f(x, t)=0\}$. In fact, this is nothing but GaussGreen's theorem expressed in the language of distributions [17, Thm. 3.1.9]. Equation (24) follows from this formula once $d s$ has been expressed in terms of $d s_{t}$ and $d t$. This is a local argument; for any $\left(x_{0}, t_{0}\right) \in \Gamma$ there exists an index $1 \leq i \leq n$ such that $\partial f\left(x_{0}, t_{0}\right) / \partial x_{i} \neq 0$, and if $d \hat{x}_{i}=d x_{1} \ldots d x_{i-1} d x_{i+1} \ldots d x_{n}$ then the identities

$$
d s=\frac{|\nabla f|}{\left|\partial f / \partial x_{i}\right|} d \hat{x}_{i} d t \quad \text { and } \quad d s_{t}=\frac{\left|\nabla_{x} f\right|}{\left|\partial f / \partial x_{i}\right|} d \hat{x}_{i}
$$

hold in some neighbourhood $U$ of $\left(x_{0}, t_{0}\right)$. Since $N=\left(\nabla_{x} f, \partial f / \partial t\right) /|\nabla f|$ we have

$$
\partial_{t} \chi=-N_{t} d s=-\frac{\partial f / \partial t}{|\nabla f|} d s=-\frac{\partial f / \partial t}{\left|\partial f / \partial x_{i}\right|} d \hat{x}_{i} d t=-\frac{\partial f / \partial t}{\left|\nabla_{x} f\right|} d s_{t} d t, \quad(\text { in } U),
$$

completing the proof, because the point $\left(x_{0}, t_{0}\right)$ was arbitrary.

Observe that $\chi_{t} \in L^{2}$ because $B_{t}$ is bounded, thus $\chi_{t}$ may be considered as an element of $H^{-1}$. Moreover, it is not difficult to show that the mapping 
$[0, T] \ni t \mapsto \chi_{t} \in H^{-1}$ is (norm-)continuous, using e.g. dominated convergence. A little more difficult is the following:

Lemma 8 For any $t \in[0, T]$ the surface measure $d s_{t}$ belongs to $H^{-1}$, and the mapping $[0, T] \ni t \mapsto d s_{t} \in H^{-1}$ is continuous.

PROOF. There exists a vector field $\nu=\left(\nu^{1}, \ldots, \nu^{n}\right) \in C_{0}^{1}\left(\mathbf{R}^{n} \times[0, T] ; \mathbf{R}^{n}\right)$ such that $\nu(x, t)$ restricted to $\Gamma_{t}$ coincides with the exterior unit normal to $B_{t}$ for all $t \in[0, T]$. In fact, since $\Gamma=\{(x, t): f(x, t)=0\}$ is compact, there exists a bounded open neighbourhood $U$ of $\Gamma$, so that the vector field

$$
\tilde{\nu}(x, t)=\frac{\nabla_{x} f(x, t)}{\left|\nabla_{x} f(x, t)\right|}
$$

is continuously differentiable on $U$. If $\varphi \in C_{0}^{1}(U)$ equals one in a neighbourhood of $\Gamma$, then $\nu=\varphi \tilde{\nu}$ has the desired properties. Now, if $u \in C_{0}^{1}\left(\mathbf{R}^{n}\right)$, and use the notation $\nu_{t}(x)=\nu(x, t)$, then an application of the divergence theorem gives the following representation for the surface measure:

$$
\int_{\Gamma_{t}} u d s_{t}=\int_{\Gamma_{t}} u \nu_{t} \cdot n d s_{t}=\int_{B_{t}} \nabla \cdot\left(u \nu_{t}\right) d x=\int_{B_{t}}\left(\nabla u \cdot \nu_{t}+u \nabla \cdot \nu_{t}\right) d x
$$

Clearly $\left|\int_{\Gamma_{t}} u d s_{t}\right| \leq C_{t}\|u\|_{H_{0}^{1}}$, where $C_{t}=\left(\sum_{i=1}^{n}\left\|\nu_{t}^{i}\right\|_{L^{2}}^{2}+\left\|\partial_{i} \nu_{t}^{i}\right\|_{L^{2}}^{2}\right)^{1 / 2}$, which proves the first assertion. The second assertion follows from (25) and the observation that $\nu_{s}^{i} \rightarrow \nu_{t}^{i}$ and $\partial_{i} \nu_{s}^{i} \rightarrow \partial_{i} \nu_{t}^{i}$ in $L^{2}$ as $s \rightarrow t$ for $i=1, \ldots, n$ and all $t \in[0, T]$.

Lemma $9[0, T] \ni t \mapsto \chi_{t} \in H^{-1}$ is (continuously) w-differentiable with wderivative

$$
\frac{d}{d t} \chi_{t}=-\frac{\partial f / \partial t}{\left|\nabla_{x} f\right|} d s_{t}
$$

PROOF. Let $\phi \in C_{0}^{\infty}\left(\mathbf{R}^{n}\right)$ be an arbitrary test function. It suffices to prove that $t \mapsto\left\langle\phi, \chi_{t}\right\rangle$ is differentiable on $(0, T)$ with derivative

$$
\frac{d}{d t}\left\langle\phi, \chi_{t}\right\rangle=\left\langle\phi,-\frac{\partial f / \partial t}{\left|\nabla_{x} f\right|} d s_{t}\right\rangle
$$

Since $(\partial f / \partial t) /\left|\nabla_{x} f\right|$ is continuous and supp $d s_{t}$ compact, Lemma 8 implies that the right hand side of (26) in continuous $[0, T] \rightarrow H^{-1}$. Therefore, it is enough to show that the identity holds in the sense of distributions. Let $\psi \in C_{0}^{\infty}(0, T)$, then it follows from Fubini's theorem, and the definition tensor 
products of distributions, that

$$
\begin{aligned}
\int_{0}^{T}-\psi^{\prime}(t)\left\langle\phi, \chi_{t}\right\rangle d t & =\int_{0}^{T} \int_{\mathbf{R}^{n}}-\psi^{\prime}(t) \phi(x) \chi(x, t) d x d t \\
& =\left\langle-\partial_{t}(\phi \otimes \psi), \chi_{t}\right\rangle \\
& =\left\langle\phi \otimes \psi, \partial_{t} \chi\right\rangle \\
& =\left\langle\phi \otimes \psi,-\frac{\partial f / \partial t}{\left|\nabla_{x} f\right|} d s_{t} d t\right\rangle=\int_{0}^{T} \psi(t)\left\langle\phi,-\frac{\partial f / \partial t}{\left|\nabla_{x} f\right|} d s_{t}\right\rangle d t
\end{aligned}
$$

where Lemma 7 was invoked in the second last equality.

The next goal is to show that $t \mapsto p_{t}$ a continuous map $[0, T] \rightarrow H_{0}^{1}$. We first compute the Laplacian of $\bar{p}_{t}$ in the sense of distributions. Let $\phi \in C_{0}^{\infty}\left(\mathbf{R}^{n}\right)$, then application of the Gauss-Green formula gives

$$
\begin{aligned}
\left\langle-\Delta \bar{p}_{t}, \phi\right\rangle & =\left\langle\bar{p}_{t},-\Delta \phi\right\rangle \\
& =-\int_{B_{t}} p_{t} \Delta \phi d x \\
& =\int_{B_{t}} \nabla p_{t} \cdot \nabla \phi d x=\int_{\Gamma_{t}} \phi \frac{\partial p_{t}}{\partial n} d s_{t}-\int_{B_{t}} \phi \Delta p_{t} d x
\end{aligned}
$$

Now, $-\Delta p_{t}=1-p_{t}$ in $B_{t}$ by (13), $\partial p_{t} / \partial n=(\partial f / \partial t) /\left|\nabla_{x} f\right|$ by $(14)$, and $\bar{p}_{t}=\chi_{t} \bar{p}_{t}$, so $\bar{p}_{t}$ satisfies the following equation

$$
(-\Delta+1) \bar{p}_{t}=\chi_{t}+\frac{\partial f / \partial t}{\left|\nabla_{x} f\right|} d s_{t},
$$

where derivatives are computed in the sense of distributions.

Lemma 10 The mapping $[0, T] \ni t \mapsto \bar{p}_{t} \in H_{0}^{1}$ is continuous.

PROOF. The identity (27) holds as an equation in $H^{-1}$. Indeed, the left hand side of the identity is clearly a member of $H^{-1}$ because $\bar{p}_{t} \in H_{0}^{1}$. Moreover, since $(\partial f / \partial t) /\left|\nabla_{x} f\right|$ is continuous on $\Gamma_{t}$ it follows from Lemma 8 , and the remark before the lemma, that the right hand side of the identity also belongs to $H^{-1}$ and depends continuously on $t$. The result now follows because $-\Delta+1$ : $H_{0}^{1} \rightarrow H^{-1}$ is an isomorphism.

PROOF of Theorem 6. We have to verify that $u_{t}$ given by (23) satisfies the conditions (21a), (21b) and (21c) of Definition 5. Clearly $\left\langle\bar{p}_{t}, \phi\right\rangle \geq 0$ for all $0 \leq \phi \in C_{0}^{\infty}\left(\mathbf{R}^{n}\right)$ so $(21 b)$ holds, by the definition of the vector-valued integral. Now, $\left\langle\bar{p}_{t}, \eta-\chi_{t}\right\rangle=0$ for any cut-off function $\eta \geq \chi_{t}$. Since $\chi_{s} \subset \chi_{t}$ for $s \leq t$ (c.f. the third remark after Definition 1) we get $\left\langle\bar{p}_{s}, \eta-\chi_{t}\right\rangle=0$, which 
implies (21c). To prove the remaining condition, observe that (27) combined with Proposition 9 yields

$$
(-\Delta+1) \bar{p}_{t}=\chi_{t}-\frac{d}{d t} \chi_{t}
$$

Applying the continuous operator $-\Delta+1: H_{0}^{1} \rightarrow H^{-1}$ to $u_{t}$ gives

$$
\begin{aligned}
(-\Delta+1) u_{t} & =\int_{0}^{t} \mathrm{e}^{t-s}(-\Delta+1) \bar{p}_{s} d s \\
& =\int_{0}^{t} \mathrm{e}^{t-s}\left(\chi_{s}-\frac{d}{d s} \chi_{s}\right) d s \\
& =\int_{0}^{t} \frac{d}{d s}\left(-\mathrm{e}^{t-s} \chi_{s}\right) d s=\mathrm{e}^{t} \chi_{0}-\chi_{t}
\end{aligned}
$$

which proves (21a) because the fundamental theorem of calculus is valid for vector-valued integrals of w-differentiable functions.

Remarks: 1 . The proof shows that the "weight" $\mathrm{e}^{t-s}$ in the Baiocchi transform (23) is an integrating factor for the right-hand side of (28).

2. The biofilm model can be generalized slightly by allowing the nutrient concentration outside the biofilm to vary with time. This leads to a problem similar to $(\mathrm{P})$ but with the pressure $p_{t}$ satisfying $(-\Delta+1) p_{t}=c(t)$ in $B_{t}$, instead of the PDE in (1). This new problem can be handled with the same methods as (P). For instance, instead of (28) a classical solution of the new problem satisfies

$$
(-\Delta+1) \bar{p}_{t}=c(t) \chi_{t}-\frac{d}{d t} \chi_{t}
$$

and, keeping in mind the first remark, we see that a result similar to Theorem 6 holds if the generalized weighted Baiocchi transform $u_{t}=\int_{0}^{t} \mathrm{e}^{C(t)-C(s)} \bar{p}_{s} d s$ is used, where $C(t)$ is a primitive function of $c(t)$.

\section{Reformulation as a Variational Inequality and Existence}

In this section it is shown that every weak solution of $(\mathrm{P})$ can be characterized, through $u_{t}$, as the solution of a family of variational inequalities.

One cannot simply solve for $u_{t}$ in (21a), and take $\chi_{t}$ as the characteristic function of the set $\left\{x: u_{t}(x)>0\right\}$, to get a weak solution $t \mapsto \chi_{t}$ of $(\mathrm{P})$ because $\chi_{t}$ enters as an unknown on the right hand side of the equation. The seemingly radical solution to this dilemma is to throw away the unwieldy term $\chi_{t}$ in (21a)! 
More precisely, suppose $[0, T] \ni t \mapsto \chi_{t} \in \mathcal{X}$ is a weak solution of $(\mathrm{P})$, fix a time $t, 0<t \leq T$, and let $\eta$ be a cutoff function such that $\eta \geq \chi_{t}$. Then rewrite $(21 \mathrm{a})$ in the form

$$
(-\Delta+1) u_{t}=\rho_{t}+\eta-\chi_{t}
$$

where

$$
\rho_{t}=\mathrm{e}^{t} \chi_{0}-\eta
$$

Using the inequalities $\eta-\chi_{t} \geq 0, u_{t} \geq 0$, and combining (29) with (21c), we see that $u_{t}$ satisfies the following linear complementary problem:

$$
\begin{aligned}
(-\Delta+1) u_{t} & \geq \rho_{t}, \\
u_{t} & \geq 0, \\
\left\langle(-\Delta+1) u_{t}-\rho_{t}, u_{t}\right\rangle & =0 .
\end{aligned}
$$

It is well known that such a problem is equivalent to a variational inequality: Let $K$ be the closed convex subset of $H_{0}^{1}$ given by

$$
K=\left\{v \in H_{0}^{1}: v \geq 0 \quad \text { a.e. on } \mathbf{R}^{n}\right\}
$$

then $u_{t}$ solves the linear complementary problem (31a)-(31c) if and only if

$$
u_{t} \in K, \quad \text { and } \quad\left(u_{t}, v-u_{t}\right)_{H_{0}^{1}} \geq\left\langle\rho_{t}, v-u_{t}\right\rangle \quad \text { for all } v \in K,
$$

where $(u, v)_{H_{0}^{1}}=\int(\nabla u \cdot \nabla v+u v) d x$ is the scalar product on $H_{0}^{1}$, c.f. $\S 4$. To see this, assume first that $u_{t}$ solves the linear complementary problem. Clearly $u_{t} \in K$ by (31b). Next, observe that Equation (31c) is equivalent to the identity

$$
\left(u_{t}, u_{t}\right)_{H_{0}^{1}}-\left\langle\rho_{t}, u_{t}\right\rangle=0,
$$

by the definition of $-\Delta+1$ (see $\S 4$ ). Similarly, (31a) implies that for all $v \in K$,

$$
0 \leq\left\langle(-\Delta+1) u_{t}-\rho_{t}, v\right\rangle=\left(u_{t}, v\right)_{H_{0}^{1}}-\left\langle\rho_{t}, v\right\rangle
$$

If (33) is subtracted from this inequality, then we get the variational inequality in (32), and the assertion is proved in one direction. For the converse, assume that $u_{t}$ solves the variational inequality. Then $(31 \mathrm{~b})$ is automatically fulfilled, and by taking $v=u_{t}+w$ in (32), for any $w \in K$, it is easy to see that (31a) holds. Finally, (31c) follows if $v=2 u_{t}$ and $v=0$ are substituted into (32).

It is now easy to establish existence and uniqueness of solutions to the variational inequality:

Proposition 11 For each $t \in[0, T]$ the variational inequality (32) has a unique solution $u_{t} \in H_{0}^{1}$, and the mapping $[0, T] \ni t \mapsto u_{t} \in H_{0}^{1}$ is continuous. Moreover, $\left\|u_{t}\right\|_{H_{0}^{1}} \leq\left\|\rho_{t}^{+}\right\|_{L^{2}} \leq\left(\mathrm{e}^{t}-1\right)\left\|\chi_{0}\right\|_{L^{2}}$, and $\operatorname{supp} \rho_{t}^{+} \subset \operatorname{supp} u_{t}$, where $\rho_{t}^{+}=\max \left\{0, \rho_{t}\right\}$. 
PROOF. Existence and uniqueness for (32) is nothing but the usual projection theorem from Hilbert space theory (for projections onto closed convex sets), see Rudin [24, Theorem 4.10] or Brézis [4, Théorème V.2]. Let us briefly recall the argument: By Riesz' lemma there exists a vector $\bar{\rho}_{t} \in H_{0}^{1}$ such that $\left(\bar{\rho}_{t}, v\right)_{H_{0}^{1}}=\left\langle\rho_{t}, v\right\rangle$ for all $v \in H_{0}^{1}$. (In fact, this is the content of the identity $(-\Delta+1) \bar{\rho}_{t}=\rho_{t}$.) Then $(32)$ becomes the problem of finding a vector $u_{t} \in K$ such that

$$
\left(\bar{\rho}_{t}-u_{t}, v-u_{t}\right)_{H_{0}^{1}} \leq 0 \quad \text { for all } v \in K,
$$

which, in turn, is equivalent to that of finding $u_{t} \in K$ such that

$$
\left\|\bar{\rho}_{t}-u_{t}\right\|_{H_{0}^{1}}=\inf _{v \in K}\left\|\bar{\rho}_{t}-v\right\|_{H_{0}^{1}}
$$

The latter problem is uniquely solvable by the projection theorem. In fact, the solution is $u_{t}=P_{K} \bar{\rho}_{t}$ where $P_{K}$ denotes the projection onto the closed convex set $K$.

To prove continuity of the map $t \mapsto u_{t}$, recall that $P_{K}$ does not increase the norm, so for any $s, t \in[0, T]$,

$$
\begin{aligned}
\left\|u_{t}-u_{s}\right\|_{H_{0}^{1}} & \leq\left\|\bar{\rho}_{t}-\bar{\rho}_{s}\right\|_{H_{0}^{1}} \\
& =\left\|\rho_{t}-\rho_{s}\right\|_{H^{-1}} \leq\left\|\chi_{0}\right\|_{L^{2}}\left|\mathrm{e}^{t}-\mathrm{e}^{s}\right| .
\end{aligned}
$$

The bound on $\left\|u_{t}\right\|_{H_{0}^{1}}$ is obtained by estimating the right-hand side of the inequality $\left\|u_{t}\right\|_{H_{0}^{1}}^{2}=\left\langle\rho_{t}, u_{t}\right\rangle \leq\left\langle\rho_{t}^{+}, u_{t}\right\rangle$, which follows from (33) because $u_{t} \geq 0$. Finally, to prove the inclusion, set $V=\left(\operatorname{supp} u_{t}\right)^{c}$, then $0=\left\langle(-\Delta+1) u_{t}, \phi\right\rangle \geq$ $\left\langle\rho_{t}, \phi\right\rangle$ for all non-negative test functions $\phi \in C_{0}^{\infty}(V)$, by (31a). This shows that $\rho_{t} \leq 0$ on $V$, hence $V \subset\left(\operatorname{supp} \rho_{t}^{+}\right)^{c}$, and the assertion follows.

We have the following regularity result:

Proposition 12 If $u_{t}$ solves the variational inequality (32) then $u_{t} \in W^{2, p}$ for all $1 \leq p<\infty$. In particular $u_{t} \in C^{1, \alpha}\left(\mathbf{R}^{n}\right)$, for all $0<\alpha<1$.

PROOF. Suppose we know that

$$
\rho_{t} \leq(-\Delta+1) u_{t} \leq \rho_{t}^{+}
$$

where $\rho_{t}^{+}=\max \left\{0, \rho_{t}\right\}$. Then $(-\Delta+1) u_{t}:=f_{t} \in L^{p}\left(\mathbf{R}^{n}\right)$ for all $1 \leq p \leq \infty$. So, by well-known properties of the Bessel potentials (see e.g. Stein [27, p. 135]), it follows that $u_{t}=(-\Delta+1)^{-1} f_{t} \in W^{2, p}$ for $1 \leq p<\infty$. Sobolev's inequalities then implies $u_{t} \in C^{1, \alpha}\left(\mathbf{R}^{n}\right)$ for any $0 \leq \alpha<1$. 
To establish (34) we adapt a nice trick from [13, Theorem 4] to our problem (See also [14].): Let a closed convex subset of $H_{0}^{1}$ be defined by

$$
K_{t}=\left\{v \in H_{0}^{1}: \rho_{t} \leq(-\Delta+1) v \leq \rho_{t}^{+}\right\},
$$

and denote by $w_{t}$ the solution of the variational inequality:

$$
w_{t} \in K_{t} \quad \text { and } \quad\left(w_{t}, v-w_{t}\right)_{H_{0}^{1}} \geq 0 \quad \text { for all } v \in K_{t} .
$$

Using an argument similar to the one in the proof of Proposition 11, it is easy to see that $w_{t}$ exists and is unique. The aim is now to prove that $w_{t}=u_{t}$, which will imply (34). This identity is achieved by showing that $w_{t}$ solves the linear complementary problem (31a)-(31c), whose unique solution is $u_{t}$. First, notice that (31a) automatically holds for $w_{t}$, by the definition of $K_{t}$. Next, use that the variational inequality (35) can be rewritten in the form

$$
\left\langle(-\Delta+1) v, w_{t}\right\rangle \geq\left\langle(-\Delta+1) w_{t}, w_{t}\right\rangle \quad \text { for all } v \in K_{t} .
$$

If $v$ is defined by the equation $(-\Delta+1) v=\rho_{t}$, then $v \in K_{t}$, and (36) becomes

$$
0 \geq\left\langle(-\Delta+1) w_{t}-\rho_{t}, w_{t}\right\rangle .
$$

Now, if we can prove $(31 \mathrm{~b})$, i.e. $w_{t} \geq 0$, then the reverse inequality follows from (31a), implying (31c), and we are finished. To prove that $w_{t} \geq 0$, set $N=\left\{x: w_{t}<0\right\}$. Then $N$ is measurable (in fact open, because $w_{t} \in C^{1, \alpha}\left(\mathbf{R}^{n}\right)$, by the argument at the beginning of the proof). Now, take $v$ in (36) to be the element of $K_{t}$ defined by

$$
(-\Delta+1) v= \begin{cases}\rho_{t}^{+} & \text {in } N \\ (-\Delta+1) w_{t} & \text { otherwise }\end{cases}
$$

Then we get

$$
0 \geq\left\langle(-\Delta+1) w_{t}-\rho_{t}^{+}, w_{t}^{-}\right\rangle
$$

where $w_{t}^{-}=\min \left(w_{t}, 0\right) \in H_{0}^{1}$. This inequality implies

$$
0 \geq\left\langle w_{t}^{-}, \rho_{t}^{+}\right\rangle \geq\left\langle(-\Delta+1) w_{t}, w_{t}^{-}\right\rangle \geq\left\|w_{t}^{-}\right\|_{H_{0}^{1}}^{2}
$$

hence $w_{t}^{-}=0$, as wanted, and the proof is complete.

We have the following comparison result, see [13, Lemma 4.1] or [3].

Proposition 13 Let $u_{t}$ be the solution of the variational inequality (32). If $v \in H_{0}^{1}$ satisfies $v \geq 0$ a.e. and $(-\Delta+1) v \geq \rho_{t}$, then

$$
u_{t}(x) \leq v(x) \quad \text { a.e. } \mathbf{R}^{n} .
$$

In particular, $u_{t}(x)=\min \left\{v(x): v \geq 0\right.$ a.e. and $\left.(-\Delta+1) v \geq \rho_{t}\right\}$. 
PROOF. Let $v$ satisfy the hypothesis in the proposition. The aim is to prove that $w=u_{t}-v \leq 0$ almost everywhere on $\mathbf{R}^{n}$. The set $D=\left\{x: u_{t}(x)>0\right\}$ is open in $\mathbf{R}^{n}$ because $u_{t}$ is continuous, by Proposition 12 , and the identity

$$
(-\Delta+1) u_{t}=\rho_{t} \quad(\text { on } D)
$$

holds in the sense of distributions. The function $w^{+}=\max (w, 0)$ is nonnegative, belongs to $H_{0}^{1}$, and $\operatorname{supp}\left(w^{+}\right) \subset D$. Since it follows from (38) that $(-\Delta+1) w \leq \rho_{t}-\rho_{t}=0$ on $D$, we therefore get the inequality

$$
0 \geq\left\langle w^{+},(-\Delta+1) w\right\rangle \geq\left\|w^{+}\right\|_{H_{0}^{1}}^{2} .
$$

Thus $w^{+}=0$, implying $w \leq 0$ a.e., as wanted. Since we may take $v$ equal to $u_{t}$, the extremal property of $u_{t}$ follows.

Definition 14 A mapping $[0, T] \ni t \mapsto u_{t} \in H_{0}^{1}\left(\mathbf{R}^{n}\right)$ is called a variational solution of $(\mathrm{P})$ with initial data $\chi_{0}$ if there exists a cut-off function $\eta \in C_{0}^{\infty}\left(\mathbf{R}^{n}\right)$, $0 \leq \eta \leq 1$, such that (i) $u_{t}$ satisfies (32) with $\rho_{t}=\mathrm{e}^{t} \chi_{0}-\eta$, and (ii) $\eta u_{t}=u_{t}$, for all $t, 0 \leq t \leq T$.

We have seen that to each weak solution $t \mapsto \chi_{t}$ there corresponds a variational solution $t \mapsto u_{t}$.

Lemma 15 Suppose $t \mapsto u_{t}, 0 \leq t \leq T$, is a variational solution of $(P)$ with initial data $\chi_{0}$ (i.e. $\left.\rho_{t}=\mathrm{e}^{t} \chi_{0}-\eta\right)$. Suppose $\eta^{*}$ is a cut-off function such that $\eta^{*} \geq \eta$, and let $u_{t}^{*}$ denotes the solution of (32) with $\rho_{t}^{*}=\mathrm{e}^{t} \chi_{0}-\eta^{*}$ in the right-hand side. Then $u_{t}^{*}=u_{t}$ for all $t \in[0, T]$.

PROOF. Since $\rho_{t}^{*} \leq \rho_{t}$ Proposition 13 implies $u_{t}^{*} \leq u_{t}$. In particular, $\eta u_{t}^{*}=$ $u_{t}^{*}$ because $\eta=1$ on $\operatorname{supp}\left(u_{t}\right)$. By definition $u_{t}^{*}$ satisfies

$$
\left(u_{t}^{*}, v-u_{t}^{*}\right)_{H_{0}^{1}} \geq\left\langle\rho_{t}^{*}, v-u_{t}^{*}\right\rangle \quad \text { for all } v \in K .
$$

Taking test functions of the form $v=\eta w, w \in K$, in this inequality gives the condition

$$
\left(u_{t}^{*}, \eta\left(w-u_{t}^{*}\right)\right)_{H_{0}^{1}} \geq\left\langle\rho_{t}^{*}, \eta\left(w-u_{t}^{*}\right)\right\rangle \quad \text { for all } w \in K .
$$

Using that $\left(u_{t}^{*}, \eta v\right)_{H_{0}^{1}}=\left(\eta u_{t}^{*}, v\right)_{H_{0}^{1}}$, for any $v \in H_{0}^{1}$, we see that $u_{t}^{*}$ must satisfy

$$
\left(u_{t}^{*}, w-u_{t}^{*}\right)_{H_{0}^{1}} \geq\left\langle\eta \rho_{t}^{*}, w-u_{t}^{*}\right\rangle \quad \text { for all } w \in K .
$$

Now, $\eta \geq \eta \eta^{*}$ implies $\eta \rho_{t}^{*} \geq \rho_{t}$, so a second application of Proposition 13 gives $u_{t}^{*} \geq u_{t}$, and the proof is complete. 
Theorem 16 For each $\chi_{0} \in \mathcal{X}$ there exists a unique mapping $\mathbf{R}_{+} \ni t \mapsto u_{t} \in$ $H_{0}^{1}$ with the following properties: (a) $t \mapsto u_{t}$ is continuous and increasing $\left(u_{t} \geq\right.$ $u_{s}$ for $\left.t \geq s\right)$. (b) For each $T>0$, the restricted mapping $[0, T] \ni t \mapsto u_{t} \in H_{0}^{1}$ is a variational solution of $(P)$ with initial data $\chi_{0}$. (c) If $\operatorname{supp} \chi_{0} \subset B\left(a ; R_{0}\right)$ for some $a \in \mathbf{R}^{n}$ and $R_{0}>0$, then $\operatorname{supp} u_{t} \subset B\left(a ; R_{0}+t\right)$ for all $t>0$.

PROOF. If $\chi_{0}^{*}=\chi_{B\left(a ; R_{0}\right)}$, then $\chi_{0}^{*} \geq \chi_{0}$. Let $u_{t}^{*}$ denote the Baiocchi transform (23) of the radial solution (18) of the problem (P). (See §3.2.) Then $t \mapsto u_{t}^{*}, t \geq 0$, is the variational solution of $(\mathrm{P})$ with initial data $\chi_{0}^{*}$, satisfying

$$
\operatorname{supp} u_{t}^{*} \subset B\left(a ; R_{0}+t\right)
$$

by Proposition 2 and Corollary 4 . Given $T>0$, choose a cut-off function $\eta_{T}$ such that $\eta_{T}=1$ in a neighbourhood of $B\left(a ; R_{0}+T\right)$, and let $t \mapsto u_{t}^{T}$ be the solution of (32) with $\rho_{t}=\mathrm{e}^{t} \chi_{0}-\eta_{T}$. By Proposition 13, $u_{t}^{T} \leq u_{t}^{*}$ for all $t$, hence

$$
\operatorname{supp} u_{t}^{T} \subset \operatorname{supp} u_{t}^{*} \subset B\left(a ; R_{0}+t\right) \text {, }
$$

so $\eta_{T} u_{t}^{T}=u_{t}^{T}$, for $0 \leq t \leq T$. Therefore $[0, T] \ni t \mapsto u_{t}^{T} \in H_{0}^{1}$ is a variational solution of $(\mathrm{P})$ with initial data $\chi_{0}$, as wanted. To define the mapping $\mathbf{R}_{+} \ni$ $t \rightarrow u_{t} \in H_{0}^{1}$, set $u_{t}=u_{t}^{T}$ for some $T>t$. The mapping is well-defined because $u_{t}$ is independent of the choice of $T$, by Lemma 15. Continuity follows from Proposition 11, and monotonicity from Proposition 13, because $\rho_{t} \geq \rho_{s}$ for $t \geq s$.

\section{From Variational to Weak Solutions}

In this section it is shown that every variational solution of $(\mathrm{P})$ is also a weak solution of $(\mathrm{P})$, so that the two notions of solvability are in fact equivalent.

Theorem 17 Suppose $[0, T] \ni t \mapsto u_{t} \in H_{0}^{1}$ is a variational solution of $(P)$ with initial data $\chi_{0} \in \mathcal{X}$. For $0<t \leq T$, define $\chi_{t}$ as the characteristic function of the set $B_{t}=\left\{x: u_{t}(x)>0\right\}$, and set $\chi_{t}=\chi_{0}$ when $t=0$. Then the mapping $[0, T] \ni t \mapsto \chi_{t} \in \mathcal{X}$ is a weak solution of $(P)$.

PROOF. Since $u_{t} \in C_{0}^{1}\left(\mathbf{R}^{n}\right)$ by Proposition 12 and Theorem 16, the sets $B_{t}$ clearly belongs to $\mathcal{B}$, for $0<t \leq T$, so $t \mapsto \chi_{t}$ is a well-defined mapping from $[0, T]$ to $\mathcal{X}$. We have to show that $u_{t}$ satisfies the equations $(21 \mathrm{a})-(21 \mathrm{c})$ in Definition 5. First of all, observe that

$$
(-\Delta+1) u_{t}-\rho_{t}=0 \quad \text { on } B_{t}
$$


Since $u_{t} \equiv 0$ on the complement of $B_{t}$ and $u_{t} \in H^{2}$ (Proposition 12 again) it follows that

$$
(-\Delta+1) u_{t}=0 \quad \text { on } \mathbf{R}^{n} \backslash B_{t} .
$$

Combining the above identities proves (21a):

$$
(-\Delta+1) u_{t}=\chi_{t} \rho_{t}=\chi_{t}\left(\mathrm{e}^{t} \chi_{0}-\eta\right)=\mathrm{e}^{t} \chi_{0}-\chi_{t} \quad \text { on } \mathbf{R}^{n}
$$

because $\chi_{t} \geq \chi_{0}$ (by the last statement in Proposition 11), and the cut-off function $\eta$ equals one the support of $u_{t}$. Since (21b) and (21c) are obvious, the proof is complete.

As a consequence of the above theorem we immediately obtain:

Corollary 18 For each $B_{0} \in \mathcal{B}$ there exists a unique mapping $\mathbf{R}_{+} \ni t \mapsto \chi_{t} \in$ $\mathcal{X}$ with the following properties: (a) $\chi_{0}=\chi_{B_{0}}$. (b) $t \mapsto \chi_{t}$ is increasing. (c) For any $T>0$, the restricted map $[0, T] \ni t \mapsto \chi_{t} \in \mathcal{X}$ is a weak solution of (P) with initial data $\chi_{B_{0}}$. (d) If $B_{0} \subset B\left(a ; R_{0}\right)$ for some $a \in \mathbf{R}^{n}$ and $R_{0}>0$, then $\chi_{t} \leq \chi_{B\left(a ; R_{0}+t\right)}$ for all $t>0$.

Remark: Having shown equivalence between the notions of weak and variational solutions, we see that $(\mathrm{P})$ is what Crank $[6, \S 2.12]$ refers to as a degenerate free-boundary problem; the moving boundary $\Gamma_{t}$ can be determined at any time $t$, by solving a single elliptic variational inequality of the type (32), using e.g. the PSOR-algorithm (c.f. [6, p. 356]), without the need to know the solution at earlier times. However, due to the exponential weight the dataterm $\rho_{t}=\mathrm{e}^{t} \chi_{0}-\eta$ in (32), the corresponding numerical problem may be ill-conditioned when $t$ is large. It may therefore be advantageous to divide the time-interval $[0, T]$ into $N$ (possibly large) time steps $\Delta t$ (i.e. $T=N \Delta t$ ), and successively compute $\chi_{k \Delta t}, k=1, \ldots, N$, by using previous set, $\chi_{(k-1) \Delta t}$, as initial data in (32), as illustrated in Figure 2. That this yields the correct result follows from the next theorem.

The class of weak solutions of $(\mathrm{P})$ forms a semi-group. Before we can prove this, the initial data needs to incorporate into the notation: For any $B_{0} \in \mathcal{B}$ let the weak solution of $(\mathrm{P})$ with initial data $\chi_{B_{0}}$ be denoted $t \mapsto \chi_{t}\left(B_{0}\right)$, and the corresponding variational solution (see Definition 5 ) by $t \mapsto u_{t}\left(B_{0}\right)$.

Theorem 19 Suppose $\mathbf{R}_{+} \ni t \mapsto \chi_{t}\left(B_{0}\right) \in \mathcal{X}$ is the weak solutions of $(P)$ with initial data $\chi_{B_{0}}$. Define $B_{t} \in \mathcal{B}$ by $\chi_{t}\left(B_{0}\right)=\chi_{B_{t}}$. Then $\chi_{r}\left(B_{t}\right)=\chi_{t+r}\left(B_{0}\right)$ for all $r, t \geq 0$. In particular,

$$
u_{t+r}\left(B_{0}\right)=\mathrm{e}^{r} u_{t}\left(B_{0}\right)+u_{r}\left(B_{t}\right) \quad \text { for all } r, t \geq 0,
$$

where $\mathbf{R}_{+} \ni t \mapsto u_{t}\left(B_{0}\right) \in H_{0}^{1}$ denotes the corresponding variational solution of $(P)$. 


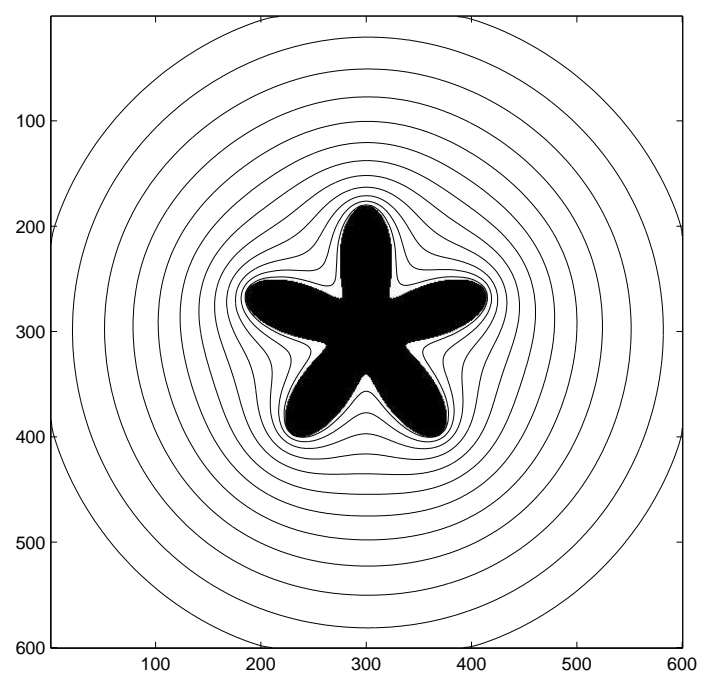

Fig. 2. A solution to the moving-boundary problem (P) obtained by solving the linear complementary problem (31a)-(31c) using the PSOR-algorithm. The initial biofilm colony $B_{0}$ is the star-shaped region shown in black, and the surrounding closed curves correspond to the biofilm interfaces $\Gamma_{t}$ at time $t=k \Delta t$ where $k=1, \ldots, 12$ and the time step is $\Delta t=0.25$.

PROOF. For $t \geq 0$ fixed, we claim that the mapping $s \mapsto \chi_{s}^{*}$ defined by

$$
\chi_{s}^{*}=\left\{\begin{array}{l}
\chi_{s}\left(B_{0}\right), \quad 0 \leq s<t, \\
\chi_{s-t}\left(B_{t}\right), t \leq s<\infty
\end{array}\right.
$$

is a weak solution of $(\mathrm{P})$ with initial data $\chi_{B_{0}}$. To prove this, we define $s \mapsto u_{s}^{*}$ by

$$
u_{s}^{*}= \begin{cases}u_{s}\left(B_{0}\right), & 0 \leq s<t, \\ \mathrm{e}^{s-t} u_{t}\left(B_{0}\right)+u_{s-t}\left(B_{t}\right), & t \leq s<\infty,\end{cases}
$$

and verify that mappings $s \mapsto \chi_{s}^{*}$, and $s \mapsto u_{s}^{*}, 0 \leq s \leq T$, satisfy the equations (21a)-(21c) in Definition 5 for all $T>0$. Clearly $u_{s}^{*} \geq 0$, so (21b) holds. An easy calculation shows that $(-\Delta+1) u_{s}^{*}=\mathrm{e}^{s} \chi_{B_{0}}-\chi_{s}^{*}$, hence (21a) holds as well. Finally, let $\eta$ be any cut-off function such that $\eta \geq \chi_{T}^{*}$. Equation (21c) holds trivially on the interval $0 \leq s<t$, so assume that $t \leq s \leq T$. Using monotonicity, $\chi_{s}^{*}=\chi_{s-t}\left(B_{t}\right)=\chi_{0}\left(B_{t}\right) \geq \chi_{t}\left(B_{0}\right)$, we find that

$$
\begin{aligned}
0 \leq\left\langle u_{s}^{*}, \eta-\chi_{s}^{*}\right\rangle & =\mathrm{e}^{s-t}\left\langle u_{t}\left(B_{0}\right), \eta-\chi_{s}^{*}\right\rangle+\left\langle u_{s-t}\left(B_{t}\right), \eta-\chi_{s}^{*}\right\rangle \\
& \leq \mathrm{e}^{s-t}\left\langle u_{t}\left(B_{0}\right), \eta-\chi_{t}\left(B_{0}\right)\right\rangle+\left\langle u_{s-t}\left(B_{t}\right), \eta-\chi_{s-t}\left(B_{t}\right)\right\rangle=0,
\end{aligned}
$$

hence (21c) holds on all of $[0, T]$. This proves the claim because $T$ was arbitrary. By the uniqueness of weak solutions, we find that $\chi_{s}^{*}=\chi_{s}\left(B_{0}\right)$ and $u_{s}^{*}=u_{s}\left(B_{0}\right)$ for all $s \geq 0$, which imply the assertions of the theorem. 


\section{Remarks About Zero Order Kinetics}

If the linear kinetics (c.f. $\left(\mathrm{H}_{2}\right)$ in $\left.§ 2.2\right)$ is replaced by zero order kinetics, $f(c)=a$, where $a>0$ constant, then we get a moving-boundary problem similar to $(\mathrm{P})$ except that the operator $-\Delta+1$, in (6), is replaced by the Laplacian $-\Delta$. This new problem, referred to as $\left(\mathrm{P}_{0}\right)$, is in some respect easier to analyze than $(\mathrm{P})$.

The definition of a classical solution of $\left(\mathrm{P}_{0}\right)$ is the same as in Definition 1, with $-\Delta+1$ replaced by $-\Delta$, of course. The classical solution corresponding to the initial set $B_{0}=\left\{x \in \mathbf{R}^{n}:|x|<R_{0}\right\}$ is radially symmetric, that is, $t \mapsto B_{t}=\{x:|x|<R(t)\}$ for some function $R=R(t)>0$. In fact, it is easy to verify that the pressure in $B_{t}$ is given by

$$
p(x)=\frac{1}{2 n}\left(R(t)^{2}-|x|^{2}\right), \quad(0 \leq|x| \leq R(t)) .
$$

It follows from the moving-boundary condition (14) that $R^{\prime}(t)=R(t) / n$, hence $R(t)=R_{0} \mathrm{e}^{t / n}$, so in contrast to $(\mathrm{P})$ the radial solutions of $\left(\mathrm{P}_{0}\right)$ grow exponentially. This means that the radial solutions satisfy

$$
\left|B_{t}\right|=\mathrm{e}^{t}\left|B_{0}\right|, \quad(t \geq 0)
$$

where $|D|$ denotes the Lebesgue measure of a measurable set $D$.

Now $-\Delta$ is not an isomorphism $H_{0}^{1} \rightarrow H^{-1}$, so in order to define weak solutions of $\left(\mathrm{P}_{0}\right)$ we follow [13], and consider the problem in the space $H_{0}^{1}(\Omega)$, where $\Omega \subset \mathbf{R}^{n}$ is a suitable bounded open set, for instance a large ball:

Definition 20 A mapping $[0, T] \ni t \mapsto \chi_{t} \in \mathcal{X}(\Omega)$ is called a weak solution of $\left(\mathrm{P}_{0}\right)$ with initial data $\chi_{0}$ if the vector-valued function $[0, T] \ni t \mapsto u_{t} \in H_{0}^{1}(\Omega)$, where $u_{t}$ is defined by the equation

$$
-\Delta u_{t}=\mathrm{e}^{t} \chi_{0}-\chi_{t}, \quad \text { (equality in } H^{-1}(\Omega) \text { ) }
$$

satisfies the conditions, $u_{t} \geq 0$ and $\left\langle u_{t}, 1-\chi_{t}\right\rangle=0$. for all $t \in[0, T]$.

Remark. This time $1 \in H^{-1}(\Omega)$, because $\Omega$ is bounded, so cut-off functions are not needed.

Now, if $t \rightarrow B_{t}$ is a classical solution of $\left(\mathrm{P}_{0}\right)$ defined for $0 \leq t \leq T$, then a result analogous to Theorem 6 holds (if $\Omega$ is chosen sufficiently large) : If $u_{t}$ is the Baiocchi transform (23) of the extended pressure $\bar{p}_{t}$ corresponding to radial solution $t \mapsto B_{t}$, then $t \mapsto \chi_{t}:=\chi_{B_{t}}$ is a weak solution of $\left(\mathrm{P}_{0}\right)$, represented by $t \mapsto u_{t}$. The proof is similar to that for $(\mathrm{P})$. It can also be shown, by a suitable modification of the proofs in $\S 6$, that each of the functions $u_{t}, 0 \leq t \leq T$, 
solves a variational inequalities of the form:

$$
\left\{\begin{array}{l}
u_{t} \in K=\left\{v \in H_{0}^{1}(\Omega): v \geq 0\right\} \\
\left(\left(u_{t}, v-u_{t}\right)\right)_{H_{0}^{1}(\Omega)} \geq\left\langle\mathrm{e}^{t} \chi_{0}-1, v-u_{t}\right\rangle \text { for all } v \in K
\end{array}\right.
$$

where $((u, v))_{H_{0}^{1}(\Omega)}=\int_{\Omega} \nabla u \cdot \nabla v d x$. Conversely, the variational solution of $\left(\mathrm{P}_{0}\right)$, obtained by solving (44) yields a weak solution of $\left(\mathrm{P}_{0}\right)$ given by $t \mapsto$ $\chi_{t}:=\chi_{B_{t}}$, where $B_{t}:=\left\{x \in \Omega: u_{t}(x)>0\right\}$. Proceeding as in [13] (with an increasing sequence of sets $\Omega$ instead of cut-off functions) it can be shown that the above weak solutions can be defined for all positive times.

Finally, the identity (42) holds for all weak solutions of $\left(\mathrm{P}_{0}\right)$. In fact, since $u_{t} \in C_{0}^{1}(\Omega)$ (because $u_{t}$ has compact support and is continuously differentiable, by a regularity result similar to Theorem 12) it follows from (43) that

$$
\mathrm{e}^{t}\left|B_{0}\right|-\left|B_{t}\right|=\int_{B} \mathrm{e}^{t} \chi_{0}-\chi_{t} d x=\int_{B}-\Delta u_{t} d x=\int_{\partial B}-\frac{\partial u_{t}}{\partial n} d s=0,
$$

where $B$ is any ball containing $\operatorname{supp} u_{t}$.

\section{A Proof of Proposition 3}

The functions $G_{\nu}(z)=(i z)^{-\nu} J_{\nu}(i z)$ (c.f. (17)) play an important role in the solution of many problems with radial symmetry, in which case $z=r \geq 0$. Recall Lommel's integral representation of the Bessel function $J_{\nu}(z)$ (Watson [31, p. 38]):

$$
J_{\nu}(z)=\frac{\left(\frac{1}{2} z\right)^{\nu}}{\Gamma\left(\nu+\frac{1}{2}\right) \Gamma\left(\frac{1}{2}\right)} \int_{0}^{\pi} \cos (z \cos \theta) \sin ^{2 \nu} \theta d \theta .
$$

If we set $z=i r$, make the change of variables $t=\cos \theta$, and employ symmetry, then we find the formulas:

$$
G_{\nu}(r)=\frac{\left(\frac{1}{2}\right)^{\nu}}{\Gamma\left(\nu+\frac{1}{2}\right) \Gamma\left(\frac{1}{2}\right)} \int_{-1}^{1}\left(1-t^{2}\right)^{\nu-\frac{1}{2}} \mathrm{e}^{ \pm r t} d t .
$$

The integrals are convergent for all $r \in \mathbf{R}$ if $\operatorname{Re} \nu>\frac{1}{2}$, and are real-valued if in addition $\nu \in \mathbf{R}$. Thus $G_{\nu}$ is essentially the Laplace transform of the $L^{1}$-function $f(t)=\left(1-t^{2}\right)_{+}^{\nu-\frac{1}{2}}$. Therefore the assertion of Proposition 3 is a consequence of the following more general result:

Theorem 21 Suppose $f \in L^{1}(\mathbf{R})$ is a non-negative almost everywhere and has compact support (in the sense of distributions), and let $F(r)$ be defined by the integral

$$
F(r)=\int_{-\infty}^{\infty} f(s) \mathrm{e}^{r s} d s, \quad(r \in \mathbf{R})
$$


If $f$ is not identically zero, then the function $F^{\prime}(r) / F(r)$ is strictly increasing on $\mathbf{R}$. (In particular, $F(r)$ is strictly logarithmically convex.) Moreover, if $[a, b]$ is the smallest interval which contains $\operatorname{supp} f$, then

$$
\lim _{r \rightarrow \infty} F^{\prime}(r) / F(r)=b \quad \text { and } \quad \lim _{r \rightarrow-\infty} F^{\prime}(r) / F(r)=a .
$$

PROOF. Define $w(r ; t)=f(t) \mathrm{e}^{r t} / F(r)$. For each $r \in \mathbf{R}, w(r ; \cdot)$ may be regarded as a weight function because $w(r ; t) \geq 0, \operatorname{supp} w(r ; \cdot) \subset[a, b]$, and $\int_{-\infty}^{\infty} w(r ; t) d t=1$. Differentiation under the integral sign in (A.2) gives

$$
\frac{F^{\prime}(r)}{F(r)}=\int_{-\infty}^{\infty} t w(r ; t) d t
$$

so clearly $a<F^{\prime}(r) / F(r)<b$. Differentiating once more yields

$$
\frac{d}{d r} \frac{F^{\prime}(r)}{F(r)}=\int_{-\infty}^{\infty} t^{2} w(r ; t) d t-\left[\int_{-\infty}^{\infty} t w(r ; t) d t\right]^{2}>0
$$

by Schwarz' inequality (or by Jensen's). Equality in this estimate would imply that $t=1$ on $\operatorname{supp} w(r ; t)$ which is impossible. Thus $F^{\prime}(r) / F(r)$ is strictly increasing on $\mathbf{R}$, and it only remains to determine its limits at infinity. In order to compute $\lim _{r \rightarrow \infty} F^{\prime}(r) / F(r)$, observe that the definition of $b$ implies $\int_{b-\epsilon / 2}^{\infty} f(s) d s>0$, for any $\epsilon>0$, hence

$\int_{-\infty}^{b-\epsilon} w(r ; t) d t=\frac{\int_{-\infty}^{b-\epsilon} f(t) \mathrm{e}^{r t} d t}{\int_{-\infty}^{\infty} f(s) \mathrm{e}^{r s} d s} \leq \frac{\mathrm{e}^{r(b-\epsilon)} \int_{-\infty}^{b-\epsilon} f(t) d t}{\mathrm{e}^{r(b-\epsilon / 2)} \int_{b-\epsilon / 2}^{\infty} f(s) d s} \leq \mathrm{e}^{-r \epsilon / 2} \frac{\int_{-\infty}^{b-\epsilon} f(t) d t}{\int_{b-\epsilon / 2}^{\infty} f(s) d s}$.

This shows that the mass of $w(r ; \cdot)$ on the interval $(-\infty, b-\epsilon]$ tends to zero rapidly as $r \rightarrow \infty$. For any $\epsilon>0$, and $r$ sufficiently large, it therefore follows that

$$
\begin{aligned}
0<b-\frac{F^{\prime}(r)}{F(r)} & =\int_{-\infty}^{\infty}(b-t) w(r ; t) d t \\
& =\int_{-\infty}^{b-\epsilon}(b-t) w(r ; t) d t+\int_{b-\epsilon}^{\infty}(b-t) w(r ; t) d t \\
& \leq(b-a) \int_{-\infty}^{b-\epsilon} w(r ; t) d t+\epsilon \int_{b-\epsilon}^{\infty} w(r ; t) d t \leq \epsilon,
\end{aligned}
$$

proving the first limit in (A.3). The second limit can be verified similarly.

Remark: Since $G_{\nu}^{\prime}(r) / G_{\nu}(r)=J_{\nu+1}(i r) / i J_{\nu}(i r)$ the limits in Proposition 3 follow form the well-known asymptotic formulas for the Bessel functions [31, Chapter VII]. However, this approach does not give us the strict monotonicity of $G_{\nu}^{\prime}(r) / G_{\nu}(r)$. 


\section{Acknowledgements}

The author wishes to thank Björn Gustafsson at the Royal Institute of Technology, Stockholm, and Stefan Diehl and professor Anders Melin at Lund University for helpful comments and discussions.

\section{References}

[1] D. J. Acheson, Elementary Fluid Dynamics, Oxford University Press, 1990.

[2] H. Begehr and R. P. Gilbert, Hele-Shaw type flows in $\mathbf{R}^{n}$, Nonlinear Anal., 10 (1986).

[3] H. BrÉzis, Problèmes unilatéraux, J. Math. Pures Appl., 51 (1972).

[4] - Analyse fonctionelle-Theorie et applications, Dunod, Paris, 1999.

[5] J. Costerton, Overview of microbial biofilms, J. Industrial Microbiology, 15 (1995).

[6] J. Crank, Free and Moving Boundary Problems, Oxford University Press, 1984.

[7] J. Dockery And I. Klapper, Finger formation in biofilm layers, SIAM J. Appl. Math., 62 (2001), pp. 853-869.

[8] H. Eberl, D. Parker, And M. van Loosdrecht, A new dererministic spatio-temporal continuum model for biofilm development, J. Theor. Medicine, 3 (2001).

[9] M. Efendiev, H. Eberl, And S. ZeliK, Existence and longtime behavior of solutions of a nonlinear reaction-diffusion system arising in the modeling of biofilms, Nonlinear diffusive systems and related topics, RIMS Tokyo, 1258 (2002).

[10] C. M. Elliott And V. Janovský, A variational inequality approach to HeleShaw flow with a moving boundary, Proc. Roy. Soc. Edinburgh, 88A (1981), pp. 93-107.

[11] D. Gilbarg and N. S. Trudinger, Elliptic Partial Differential Equations of Second Order, Springer-Verlag, Berlin, Second revised ed., 1997.

[12] H. P. Greenspan, On the growth and stability of cell cultures and solid tumors, J. Theoret. Biol., 56 (1976), pp. 229-243.

[13] B. Gustafsson, Application of variational inequalities to a moving boundary problem for Hele Shaw flows, SIAM J. Math. Anal., 16 (1985), pp. 279-300.

[14] the obstacle problem, Nonlinear Anal., 10 (1986), pp. 1487-1490. 
[15] — Direct and inverse balayage-Some new developments in classical potential theory, Nonlinear Anal., 30 (1997), pp. 2557-2565.

[16] S. Hermanowicz, A simple 2D biofilm model yields a variety of morphological features, Mathl. Biosci., 169 (2001).

[17] L. Hörmander, The Analysis of Linear Partial Differential Operators I, Springer-Verlag, Berlin, second ed., 1980.

[18] J.-U. Kreft, C. Picioreanu, J. Wimpenny, and M. Loosdrecht, Individual-based modelling of biofilms, Microbiology, 147 (2001).

[19] J. Ockendon, S. Howison, A. Lacey, And A. Movchan, Applied Partial Differential Equations, Oxford University Press, Revised ed., 2003.

[20] C. Picioreanu, M. van Loosdrecht, and J. Heijnen, Mathematical modeling of biofilm structure with a hybrid differential-discrete cellular automaton approach, Biotechnol. Bioengng., 58 (1998).

[21] _ Discrete-differential modeling of biofilm structure, Wat. Sci. Tech., 39 (1999), pp. 115-122.

[22] Y. ReICHeLt, Moving boundary problems arising from degenerate elliptic equations, Nonlinear Anal., 27 (1996), pp. 1207-1227.

[23] S. Richardson, Hele Shaw flows with a free boundary produced by the injection of fluid into a narrow channel, J. Fluid Mech., 56 (1972), pp. 609-618.

[24] W. Rudin, Real and Complex Analysis, McGraw-Hill, New York, Third ed., 1986.

[25] — Functional Analysis, McGraw-Hill, New York, Second ed., 1991.

[26] M. SAKaI, Applications of variational inequalities to the existence theorem on quadrature domains, Trans. Amer. Math. Soc., 276 (1983), pp. 267-279.

[27] E. M. Stein, Singular Integrals and Differentiability Properties of Functions, Princeton University Press, 1970.

[28] P. Stewart, New ways to stop biofilm infections, The Lancet, 361 (2003).

[29] F. Treves, Basic Linear Partial Differential Equations, Academic Press, New York, 1975.

[30] G. Wanner and W. Gujer, A multi-species biofilm model, Biotechnol. Bioengng., 28 (1986).

[31] G. N. Watson, A Treatise on the Theory of Bessel Functions, Cambridge University Press, second ed., 1996.

[32] J. Wimpenny and R. Colasanti, A unifying hypothesis for structure of microbial biofilms based on cellular automaton models, FEMS Microbiol. Ecology, 22 (1997). 